\title{
A cross-layer optimization framework for congestion and power control in cognitive radio ad hoc networks under predictable contact
}

\author{
Long Zhang ${ }^{1 *}$ D, Fan Zhuo ${ }^{1}$ and Haitao $\mathrm{Xu}^{2}$
}

\begin{abstract}
In this paper, we investigate the cross-layer optimization problem of congestion and power control in cognitive radio ad hoc networks (CRANETs) under predictable contact constraint. To measure the uncertainty of contact between any pair of secondary users (SUs), we construct the predictable contact model by attaining the probability distribution of contact. In particular, we propose a distributed cross-layer optimization framework achieving the joint design of hopby-hop congestion control (HHCC) in the transport layer and per-link power control (PLPC) in the physical layer for upstream SUs. The PLPC and the HHCC problems are further formulated as two noncooperative differential game models by taking into account the utility function maximization problem and the linear differential equation constraint with regard to the aggregate power interference to primary users (PUs) and the congestion bid for a bottleneck SU. In addition, we obtain the optimal transmit power and the optimal data rate of upstream SUs by taking advantage of dynamic programming and maximum principle, respectively. The proposed framework can balance transmit power and data rate among upstream SUs while protecting active PUs from excessive interference. Finally, simulation results are presented to demonstrate the effectiveness of the proposed framework for congestion and power control by jointly optimizing the PLPC-HHCC problem simultaneously.
\end{abstract}

Keywords: Cognitive radio, Cross-layer optimization, Congestion control, Power control, Predictable contact

\section{Introduction}

1.1 Background and motivation

Cognitive radio (CR) [1] has been widely recognized as a critical technique to mitigate the spectrum scarcity problem and enhance the overall efficiency of spectrum usage, aiming to accommodate for the evolution of wireless systems towards 5G [2]. In a CR network (CRN), unlicensed secondary users (SUs) are allowed to opportunistically access the spectrum allocated to licensed or primary users (PUs) without interfering with the coexisting PUs. That is, the SUs do not violate the quality of service (QoS) requirements of the PUs. Most of the existing research efforts in CRNs mainly focus on the issues of the physical and media access control (MAC) layers for an infrastructure-based

\footnotetext{
* Correspondence: zhanglong@hebeu.edu.cn

${ }^{1}$ School of Information and Electrical Engineering, Hebei University of

Engineering, Handan 056038, China

Full list of author information is available at the end of the article
}

single-hop scenario, including spectrum sensing, spectrum access, and sharing techniques [3-5]. In addition, SUs can also form a multi-hop decentralized ad hoc network without the support of infrastructure. In a multi-hop cognitive radio ad hoc network (CRANET) [6], SU can access the licensed spectrum by seeking to overlay, underlay, or interweave its signal with the existing PUs' signals [7]. For the underlay approach, SUs are permitted to concurrently share the licensed spectrum with PUs while guaranteeing the power of interference and noise at the PU not beyond the interference temperature limit. In this context, the interference caused by the SUs should be controlled and mitigated through effective power control strategies. Many studies on power control for CRNs have been reported from different perspectives, such as imperfect channel knowledge [8], arbitrary input distributions [9], and social utility maximization [10]. 
In comparison with the lower layer solutions as stated before, recent work indicates that there are many new challenges towards routing problem at the network layer in multi-hop CRANETs, aiming to give more insights into the impact of spectrum uncertainty on routing strategies $[11,12]$. However, the constraints and challenges with regard to SUs including random mobility, low deployment density, and limited resource along with discontinuous spectrum availability will give rise to intermittent connectivity of links among SUs in a decentralized CRANET [13]. Clearly, stochastic link outage further has a bearing on the successful transmission of data packets between a pair of SUs. To describe effective continuous transmission of SUs, the paradigm contact has been presented from different types [14], e.g., persistent contact, ondemand contact, and scheduled contact. Conceptually, a contact can be defined as a communication opportunity during which two adjacent SUs can communicate with each other. In a scheduled contact-based CRANET, multiple contacts or the set of communication opportunities can be easily derived from the statistical data of a priori available contact. In this case, the scheduled contact can be predicted and calculated accurately.

Similar to a wireline Internet or most other traditional wireless networks, network congestion in CRNs will also occur when the offered data load exceeds the available capacity of SU due to buffer overflow caused by accumulated data packets injected from upstream SUs. This therefore leads to aggressive retransmission, queuing delay, and blocking of new flows from upstream SUs. Indubitably, congestion control policy in the transport layer is essential to balance resource load and to avoid excessive congestion. However, the conventional Transmission Control Protocol (TCP) as a congestion control mechanism via window-based or acknowledgement-triggered methods is initially designed and optimized to perform in reliable wired links with constrained bit error rates (BERs) and round trip times (RTTs) [15]. Recent study by [16] has reported that the performance of HTTP download deteriorates as much as $40 \%$ under the TCP window control in an IEEE P1900.4-based cognitive wireless system using User Datagram Protocol (UDP) and TCP transport protocols. Alternatively, to accommodate for the challenging multi-hop wireless environments, some other research efforts about congestion control techniques have been conducted from the perspective of finding methods to modify TCP protocol [17]. Unfortunately, it has been also shown that these schemes of TCP modifications and extensions cannot be applied into CRANETs because of sudden large-scale bandwidth fluctuation and periodic interruption caused by spectrum sensing and channel switching $[18,19]$.

It is also noted that the TCP congestion control is targeted to regulate the data rate of upstream SUs so that the total accumulated data load does not exceed the available capacity of SU. In principle, the link capacity between a pair of SUs depend strongly on transmit power of SU coupled with wireless channel conditions [20]. By leveraging the congestion control technique, on the one hand, the attainable data rate on a wireless link between a pair of SUs depends on the interference level, which in turn rests on power control policy. On the other hand, each $\mathrm{SU}$ is expected to increase its transmit power in order to obtain as much link capacity that each flow requires [21]. However, increasing the link capacity on one link may reduce the link capacities on other links owing to mutual interference of SUs. From the above discussions, we can see that jointly optimizing transmit power in the physical layer and data rate in the transport layer for attaining the optimal link capacity becomes highly valuable. With a joint cross-layer design, the physical layer is able to share its information and configuration about optimal transmit power with the transport layer without breaking the hierarchical structure of the traditional layered architecture [22]. This motivates us to reinvestigate the cross-layer coupling between capacity supply by power control and rate demand by rate control.

\subsection{Related works}

Congestion control in wireless multi-hop networks has been widely discussed via the NUM optimization problem maximizing the total utility, subject to some different constraints including the efficiency and fairness of resource allocation [22], heterogeneous traffic [22], lossy link [23], and multipath transmission [24]. Under the condition of outage probability caused by lossy links, another work [25] investigates the rateeffective network utility maximization problem to meet with delay-constrained data traffic requirement. However, although all of the aforementioned studies consider some realistic constraints, they apply to the traditional wireless multi-hop networks only and do not consider the spectrum uncertainty in CRNs. To the best of our knowledge, some studies on congestion control for CRNs have been reported recently, although the mainstream research effort is aimed at the problems of the physical and MAC layers. In [26], Xiao et al. developed a robust active queue management scheme to stabilize the TCP queue length at base station in an infrastructure-based CRN. By using the multiple model predictive control, the proposed scheme absorbed the disturbances caused by busty 
background traffic and capacity variation. It is found that [26] is not suitable for decentralized CRANET scenario due to a lack of centralized control and global information. Unlike the condition of infrastructure-based CRN, other studies in $[18,27,28]$ have been undertaken in multi-hop CRN scenarios. In [27], Song et al. proposed an end-to-end congestion control framework without the aid of common control channel by taking into account the non-uniform channel availability. The explicit feedback mechanism without timeouts and the timeout mechanism were also investigated. In [28], Zhong et al. presented a TCP network coding dynamic generation size adjust scheme by jointly considering network coding gain and delay. The proposed scheme can significantly reduce the retransmissions and guarantee the QoS and enhance the TCP performance. In [18], Al-Ali et al. proposed an endto-end equation-based TCP friendly rate control mechanism, which achieves rate adjustment by identifying network congestion. However, the end-to-end control policy in $[18,27]$ is ill suited for operation over wireless transmission links characterized by higher RTTs, particularly if the links present the feature of intermittent connectivity in CRANET under predictable contact. On the contrary, the hop-by-hop control reacts to congestion faster where the rates are adjusted at upstream nodes by feedback information about the congestion state of intermediate nodes.

Other recent schemes that exploit the cross-layer interaction information try to deal with congestion control problem in decentralized CRNs from a crosslayer design perspective. The objective of these schemes is to improve the overall network utility while protecting active PUs' communications from excessive interference introduced by SUs. In [29], Cammarano et al. presented a distributed cross-layer framework for joint optimization of MAC, scheduling, routing, and congestion control in CRAHNs, by maximizing the throughput of a set of multi-hop end-to-end packet flows. However, similar to [18, 27], it is not clear how good the performance of the end-to-end rate control is compared under a wireless transmission environment with higher RTTs. In [30], Nguyen et al. proposed a cross-layer framework to jointly attain both congestion and power control in OFDM-based CRNs through nonconvex optimization method. By means of the adaptation of dual decomposition technique also used by [20], the distributed algorithm was developed to obtain the global optimization. In [31], Nguyen et al. further devised an optimization framework achieving trade-off between energy efficiency and network utility maximization for CRAHNs. By adjusting transmit power, persistence probability, and data rate simultaneously via the interaction between MAC and other layers, the proposed framework can jointly balance interference, collision, and congestion among SUs However, both of the frameworks in $[30,31]$ fail to take into account the impact of predictable contact or priori available contact between any pair of SUs on overall cross-layer performance.

\subsection{Our approach and contributions}

Our work in this paper mainly focuses on a decentralized CRANET under predictable contact in that it is easy to obtain the set of communication opportunities derived from the statistical available contacts among SUs. Owing to a lack of global information to achieve the centralized schedule, the cross-layer coupling between capacity supply by power control and rate demand by rate control needs to be carried out distributively by each SU via local information. Distributed implementation for power control and rate control depends on interactive processes among competitive SUs to figure out the cross-layer coupling relationships. Moreover, the objectives of SUs to maximize their utility functions are conflicting and their decisions are interactive. Apparently, it will be far more realistic to dynamically adjust transmit power and data rate according to the current instant time in the practical dynamic environment. The reason for adopting a differential game model rather than other decentralized optimization approaches is that the differential game is a continuous time dynamic game to investigate interactive decision making over time. In a differential game, the interactions among individual players are characterized by time dependency. This is in line with the nature of dynamic spectrum environment in practical CRANET scenario. Therefore, motivated by cross-layer coupling between capacity supply by power control and rate demand by rate control, we present a cross-layer optimization framework for CRANET under predictable contact by achieving the joint congestion and power control using a differential game theoretic approach. The main contributions of this paper are summarized as follows:

- To measure the uncertainty of contact between a pair of SUs, a predictable contact model is presented by deriving the probability distribution of contact via a mathematical statistics theory. By using Shannon entropy theory, we further devise an entropy paradigm to characterize quantitatively the probability distribution of contact.

- We propose a distributed cross-layer optimization framework for hop-by-hop congestion control (HHCC) and per-link power control (PLPC) for upstream SUs. The HHCC and the PLPC problems are formulated as two 
noncooperative differential game models, by taking into account the utility function maximization and linear differential equation constraint with regard to the aggregate power interference to PUs and congestion bid for bottleneck SU.

- We convert the noncooperative differential game models for the PLPC and the HHCC problems into two dynamic optimization problems. By adopting dynamic programming and maximum principle, we obtain the optimal transmit power and the optimal data rate of upstream SUs, respectively. The cross-layer optimization framework is implemented in a distributed manner through the cross-layer coordination mechanism between capacity supply by power controller and rate demand by rate controller.

\subsection{Organization and notation}

The rest of this paper is organized as follows. We firstly describe the system model in Section 2. Then, the problem formulation is presented in Section 3. In Section 4, we derive the optimal solutions to the proposed noncooperative differential game models and propose the distributed implementation approach to construct the cross-layer optimization framework. Simulation results are provided in Section 5, followed by the conclusions in Section 6 .

Notation: $\mathcal{A}$ denotes a set, and $|\mathcal{A}|$ denotes the cardinality for any set $\mathcal{A}$. We use a boldface capital to denote vector $\mathbf{A}$ to discriminate vectors from scalar quantities. $|\cdot|$ and $\|\cdot\|$ represent the absolute value of a polynomial function and the Euclidean distance between the pair of variables, respectively. $\mathbb{E}[\cdot]$ stands for the statistical expectation operator.

\section{System model}

\subsection{Network model}

Consider an underlay multi-hop CRANET coexisting with a cellular primary network as depicted in Fig. 1, wherein PUs can send their data traffic to a primary base station (PBS) via the licensed uplink channels. We denote the set of uplink channels by $\mathscr{H}=\left\{c h_{1}\right.$, $\left.c h_{2}, \cdots, c h_{\phi}\right\}$ where $\phi$ is the number of uplink channels. The uplink channel is either occupied by PUs or unoccupied. We employ the independent and identically distributed alternating ON-OFF process to model the occupation time length of PUs in uplink channels. Specifically, the OFF state indicates the idle state where the uplink channels can be freely occupied by SUs. By performing spectrum sensing on all the uplink channels periodically, $S$ SUs leverage the OFF state to access the unoccupied uplink channels by PUs. Let $\mathcal{V}=\left\{v_{1}, v_{2}, \cdots, v_{S}\right\}$ refer to the set of $S$ SUs. Each SU is equipped with two radio transceivers. One with a cognitive radio is used to opportunistically access the uplink channels for transmissions of data packets. The other is used for exchange of control signaling. Due to the randomness of data traffic and the dynamic behavior of PUs, we assume that the licensed uplink channels are available for usage by $\mathrm{SU}$ $v_{i}$ with a probability of $\delta_{i}$, for $v_{i} \in \mathcal{V}$. Based on the aforementioned ON-OFF process, the occupancy probability of uplink channel $c h_{\xi}$ by PUs is defined as $\alpha_{\xi} /\left(\alpha_{\xi}+\beta_{\xi}\right)$ [32], where $\alpha_{\xi}$ is a probability that uplink channel $\xi$ transits from OFF to ON state, and $\beta_{\xi}$ is probability that uplink channel $\xi$ transits from ON to OFF state, for $\xi=1,2, \cdots, \phi$. It is assumed that SUs can determine the occupancy probability of uplink channels by PUs through a priori knowledge of PUs' activities and local spectrum sensing. Owing to the mutually independent occupancy probability of uplink channel $c h_{\xi}$, the probability $\delta_{i}$ of uplink channels used by SU $v_{i}$ can be expressed as:

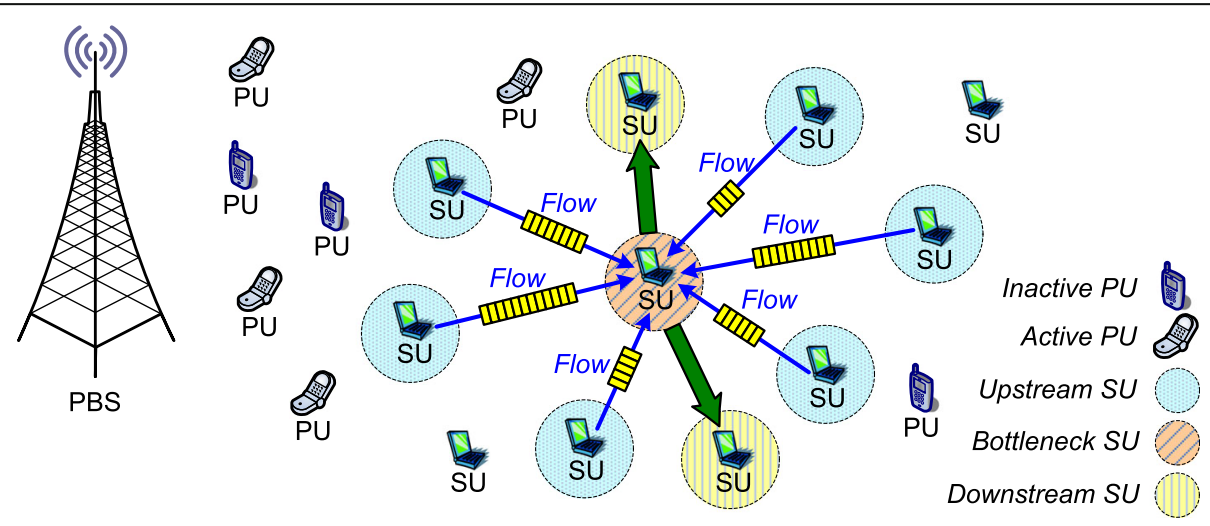

Fig. 1 Network model of the underlay multi-hop CRANET where SUs share uplink channels with PUs 


$$
\delta_{i}=\prod_{\xi=1}^{\phi}\left(1-\frac{\alpha_{\xi}}{\alpha_{\xi}+\beta_{\xi}}\right) .
$$

Different from the assumption that time is divided into fixed time slots in a discrete way, we exploit the continuous time model to represent the operation duration of the CRANET. The continuous-time operation is confined to a predefined time interval $\left[t_{0}, T\right]$. Use $\vartheta_{i}(t)$ and $z(t)$ to denote the positions of SU $v_{i}$, PU $z$ at time $t \in\left[t_{0}, T\right]$, respectively. Let $R_{T}$ and $R_{I}$ stand for the maximum transmission range of SU and the interference range of PU, respectively. Without interference with PUs, a pair of SU $v_{i}$ and $\mathrm{SU} v_{j}$ can successfully communicate with each other on channel $c h_{\xi}$ at time $t$ only if the Euclidean distance between SU $v_{i}$ and SU $v_{j}$ satisfies $\left\|\vartheta_{i}(t)-\vartheta_{j}(t)\right\| \leq R_{T}$ and when there is no any PU $z$ on channel $c h_{\mathcal{\xi}}$, i.e., $\left\|\vartheta_{i}(t)-z(t)\right\|>R_{I}$ and $\| \vartheta_{j}(t)-$ $z(t) \|>R_{I}$, for $v_{i}, v_{j} \in \mathcal{V}$ and $\xi=1,2, \cdots, \phi$. In this context, there exists a successful transmission link denoted by $l_{(i, j)}$ from SU $v_{i}$ to $\mathrm{SU} v_{j}$ on channel $c h_{\xi}$ at time $t$. For the sake of conciseness, instant time $t$ will be restricted to the time interval $\left[t_{0}, T\right]$ henceforth.

Under the constraint of successful transmission links, we assume that there are multiple different sessions from source SUs to destination SUs. Each session is associated with a route from a source SU to a destination SU. Figure 2 illustrates an example of logical topology of the underlay multi-hop CRANET, where a series of red solid line denote a session along a route from source SU1 to destination SU5, which is one of the different routes. It is assumed that a session consists of several per-link flows with elastic traffic. We use the term per-link flow to describe a sequence of data packets with elastic traffic transmitted along a successful transmission link. With regard to the route from source SU1 to destination SU5, data packets of a flow enter upstream SU2, travel via single hop, then converge at bottleneck SU3 and, finally, move to downstream SU4. We focus on a scenario that multiple different sessions converge at bottleneck SU, aiming to reinvestigate the cross-layer coupling between capacity supply by power control and rate demand by rate control at upstream SUs. From Fig. 2, the convergence of multiple flows from upstream SU2, SU6, and SU8 via single hop may result in a possible congestion at bottleneck SU3 when the offered data load exceeds the available capacity of SU3 due to a buffer overflow, although the amount of data packets has been delivered to downstream SU4, SU7, and SU9. We assume that there are $N$ flows of elastic traffic along the successful transmission links from $N$ upstream SUs to bottleneck SU $v_{b}$ via single hop, for $v_{b}$ $\in \mathcal{V}$ and $N<S$. Let $\mathcal{V}_{U P}$ and $\mathcal{N}=\{1,2, \cdots, N\}$ represent the set of $N$ upstream SUs and the set of flows of elastic traffic from $N$ upstream SUs to bottleneck SU $v_{b}$, for $\mathcal{V}_{U P} \subset \mathcal{V}$. For notational simplicity, the flow of elastic traffic along link $l_{(i, b)}$ from upstream SU $v_{i}$ to bottleneck SU $v_{b}$ is described by flow $i$, for $i \in \mathcal{N}$ and $v_{i} \in \mathcal{V}_{U P}$. Assuming that flow $i$ of elastic traffic along link $l_{(i, b)}$ arrives as a Poisson process of flow arrival intensity $\lambda_{i}$ with a size drawn independently from a common distribution of mean $\mathbb{E}\left[\lambda_{i}\right]$ [33]. When $\Psi_{(i, b)}<1$, the transmission link load, denoted by $\Psi_{(i, b)}$, induced by elastic traffic along link $l_{(i, b)}$ is equal to [33]:

$$
\Psi_{(i, b)}=\frac{\lambda_{i} \times \mathbb{E}\left[\lambda_{i}\right]}{C_{(i, b)}(\mathbf{P})},
$$

where $C_{(i, b)}(\mathbf{P})$ denotes the capacity of link $l_{(i, b)}$, and $\mathbf{P}$ $=\left\{p_{1}(t), p_{2}(t), \cdots, p_{N}(t)\right\}$ corresponds to the transmit power vector of $N$ upstream SUs at time $t$. Here, we use

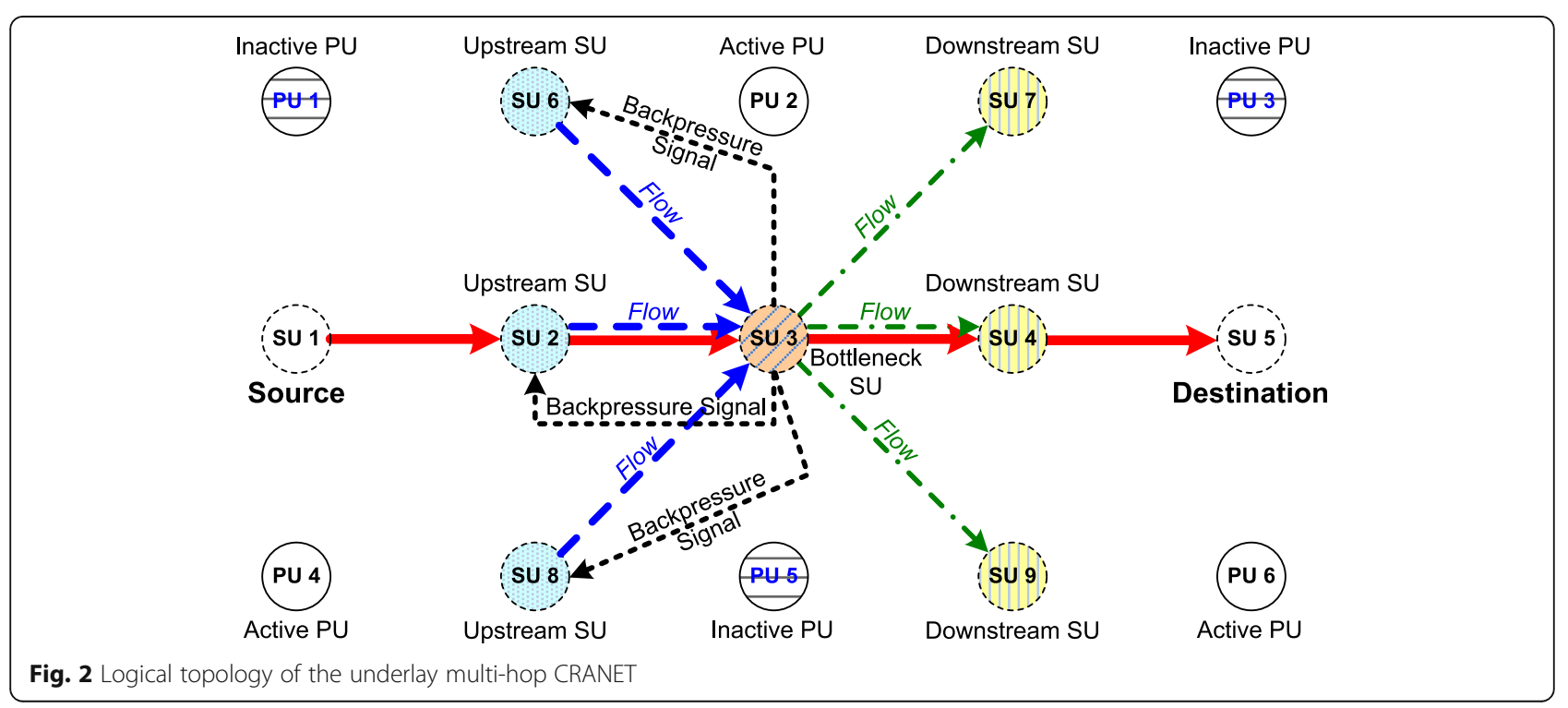


$p_{i}(t)$ to represent the instant transmit power of upstream $\mathrm{SU} v_{i}$ at time $t$. Noticing that the transmit power $p_{i}(t)$ of upstream SU $v_{i}$ can be adjusted in a continuous way but is also limited by a maximum transmit power threshold denoted by $\bar{p}_{i}$, i.e., $p_{i}(t) \in\left[0, \bar{p}_{i}\right)$. Based on elastic traffic model for each flow, the expected duration $D_{i}$ of flow $i$ with size $\mathbb{E}\left[\lambda_{i}\right]$ is given by [33]:

$$
D_{i}=\frac{\mathbb{E}\left[\lambda_{i}\right]}{C_{(i, b)}(\mathbf{P}) \times\left(1-\Psi_{(i, b)}\right)} .
$$

Under spectrum underlay scenario, SUs can simultaneously transmit with PUs but have to strictly control their transmit power to avoid interfering with coexisting PUs. Note that the simultaneous transmissions among SUs along a successful transmission link must be undertaken on the same channel, which will further incur the co-channel multiple access interference. We assume that the simultaneous transmissions among $N$ SUs along a successful transmission link on channel $c h_{\xi}$ can be undertaken under the CDMA-based medium access in the physical layer [34]. The reason for adopting the CDMA-based medium access model is that transmit power of upstream SU can be controlled to induce a different signal-to-interference-plusnoise ratio (SINR) of successful transmission link due to a co-channel multiple-access interference $[20,35,36]$. In principle, link capacity under this scenario cannot remain fixed but depends on SINR of successful transmission link between a pair of SUs. Let $\operatorname{SINR}_{(i, b)}(\mathbf{P})$ be the received SINR of bottleneck SU $v_{b}$ along link $l_{(i, b)}$ on channel $c h_{\xi}$. Therefore, the capacity of link $l_{(i, b)}$ from upstream SU $v_{i}$ to bottleneck SU $v_{b}$ can be characterized by a global and nonlinear nonconvex function of the transmit power vector and channel conditions as follows [20]:

$$
C_{(i, b)}(\mathbf{P})=\frac{1}{T_{s}} \log _{2}\left(1+\chi \cdot \operatorname{SINR}_{(i, b)}(\mathbf{P})\right)
$$

where $T_{s}$ is a symbol period and $X=-\phi_{1} / \log _{2}\left(\phi_{2}\right.$. BER) is a constant processing gain factor with $\phi_{1}$ and $\phi_{2}$ depending upon an acceptable BER along with the specific modulation and coding scheme. We assume that a large-scale slow-fading channel model is adopted to describe the line-of-sight wireless transmission environment. In this case, channel gain is subject to distance-dependent power attenuation or log-normal shadowing. As for the practical nonline-of-sight scenario, we use a Rayleigh fading model, in which the channel gain is assumed to be independent exponentially distributed random variables with unit mean [37]. Let $G_{(i, b)}$ and $F_{(i, b)}$ denote the large-scale slow-fading and the Rayleigh fading channel gain of link $l_{(i, b)}$ from upstream SU $v_{i}$ to bottleneck SU $v_{b}$, respectively. Thus, we have the normalized Rayleigh fading channel gain $\mathbb{E}\left[F_{(i, b)}\right]$ $=1$. By using the certainty-equivalent transmit power and interference power [34, 37], the received SINR of link $l_{(i, b)}$ at bottleneck SU $v_{b}$ can be expressed as:

$$
\operatorname{SINR}_{(i, b)}(\mathbf{P})=\frac{p_{i}(t) G_{(i, b)}}{I_{i}+I_{p}+n_{0}}
$$

where $n_{0}$ is the thermal noise power at bottleneck SU $v_{b}, I_{p}$ is the interference caused by the PBS, and $I_{i}$ is the aggregate power interference introduced by other upstream SUs except upstream SU $v_{i}$. The aggregate power interference is given by $I_{i}=\sum_{j \in \mathcal{N} \backslash i} p_{j}($ t) $G_{(j, b)}$. In what follows, we are targeted at the lineof-sight wireless transmission environment with the large-scale slow-fading channel gain. How to apply the dynamic fast-fading or Rayleigh fading model under the non-line-of-sight scenario into the crosslayer optimization framework for CRANET will be our further work in the future. Under spectrum underlay scenario, the interference power constraint shall be imposed to protect active PUs' communications from harmful interference caused by all the upstream SUs. We assume that the interference measurement point is located at bottleneck SU $v_{b}$ for convenience. Hence, the total interference caused by all the upstream SUs should be kept below the interference temperature limit $\varpi_{\mathrm{PBS}}$ at the interference measurement point of PBS:

$$
\sum_{i \in \mathcal{N}} p_{i}(s) G_{(i, b)} \leq \varpi_{\mathrm{PBS}}
$$

\subsection{Predictable contact model}

Considering that a contact is viewed as a communication opportunity during which two adjacent SUs can communicate with each other, we move on to model the predictable contact between a pair of SUs from a priori available contact perspective. Based on the insight into successful transmission link $l_{(i, j)}$ as noticed earlier, an encounter $e^{(i, j)}$ is defined as an effective continuous transmission between SU $v_{i}$ and $\mathrm{SU} v_{j}$ with a certain duration, for $v_{i}, v_{j} \in \mathcal{V}$. It is worth pointing out that an encounter rests on the time of incidence and the duration of an effective continuous transmission between a pair of SUs [38]. Let $t^{0 \text {, }}$ $(i, j)$ and $\Delta t^{(i, j)}$ represent the time of incidence and the duration of an encounter $e^{(i, j)}$, respectively, for 0 $<\Delta t^{(i, j)}<T-t_{0}$. Therefore, an encounter $e^{(i, j)}$ between $\mathrm{SU} v_{i}$ and $\mathrm{SU} v_{j}$ can be formulated as: 


$$
e^{(i, j)}=\left\{v_{i}, v_{j}, t^{0,(i, j)}, \Delta t^{(i, j)}\right\}
$$

Suppose that there exist $K$ encounters between $\mathrm{SU} v_{i}$ and SU $v_{j}$ within a predefined time interval $\left[t_{0}, T\right]$. In particular, the $\ell$ th encounter $e_{\ell}^{(i, j)}$ between SU $v_{i}$ and SU $v_{j}$ with a duration $\Delta t_{\ell}^{(i, j)}$, for $\ell=1,2, \cdots, K$, is given as:

$$
e_{\ell}^{(i, j)}=\left\{v_{i}, v_{j}, t_{\ell}^{0,(i, j)}, \Delta t_{\ell}^{(i, j)}\right\} .
$$

where $t_{\ell}^{0,(i, j)}$ refers to the time of incidence of the $\ell$ th encounter $e_{\ell}^{(i, j)}$. For mathematical tractability, we use the duration $\Delta t_{\ell}^{(t, j)}$ in (8) to characterize the $\ell$ th encounter, i.e., $e_{\ell}^{(i, j)} \triangleq \Delta t_{\ell}^{(i, j)}$. Thus, within time interval $\left[t_{0}, T\right]$, contact $\mathcal{C}^{(i, j)}$ between $\mathrm{SU} v_{i}$ and $\mathrm{SU} v_{j}$ can be rigorously regarded as the set of all encounters, i.e., $\mathcal{C}^{(i, j)}=\left\{e_{1}^{(i, j)}, e_{2}^{(i, j)}, \cdots, e_{K}^{(i, j)}\right\}$ and $\mid$ $\mathcal{C}^{(i, j)} \mid=K$. Note that the $\ell$ th encounter $e_{\ell}^{(i, j)}$ in contact $\mathcal{C}^{(i, j)}$ can be referred to a random variable due to the uncertainty of communication opportunity between $\mathrm{SU} v_{i}$ and SU $v_{j}$. In this way, we turn to employ a mathematical statistics theory to attain the probability distribution $\boldsymbol{Y}^{(i, j)}=\{$ $\left.\rho_{1}^{(i, j)}, \rho_{2}^{(i, j)}, \cdots, \rho_{M}^{(i, j)}\right\}$ of contact $\mathcal{C}^{(i, j)}$, which has been derived from Algorithm 1. In Algorithm 1, we introduce a coefficient $M$ to denote the number of the subintervals, which is obtained by dividing interval $[a, b]$ equally. According to the approximate derivation of the sample distribution in mathematical statistics, coefficient $M$ should be reasonably assigned, depending on the number of encounters of $K$. That is, when $K \leq 100$, coefficient $M$ can range from 5 to 12 . Obviously, it will be possible to measure the uncertainty of contact $\mathcal{C}^{(i, j)}$ between $\mathrm{SU} v_{i}$ and $\mathrm{SU} v_{j}$ by the aid of the probability distribution $Y^{(i, j)}$. Thus, by analyzing the statistical data of a priori available contact or all encounters between a pair of SUs, it is implicitly understood that the contact can be in a sense predicted very accurately.
Table 1 summarizes the constrained relationship between the number of encounters within subinterval $\left(d_{l-1}, d_{l}\right]$ and contact probability $\rho_{l}^{(i, j)}$ in Algorithm 1, for $l=1,2, \cdots, M$. It is important to emphasize that the probability distribution $\gamma^{(i, j)}$ belongs to a complete probability distribution, i.e., $\sum_{l=1}^{M} \rho_{l}^{(i, j)}=1$. Technically, the entropy paradigm is widely used for a measure of the uncertainty or randomness associated with a random variable in information theory [39]. In order to characterize quantitatively the probability distribution $\gamma^{(i, j)}$, we put forward an entropy paradigm by using Shannon entropy theory to measure the uncertainty of contact $\mathcal{C}^{(i, j)}$. Specifically, the entropy $H\left(\mathcal{Y}^{(i, j)}\right)$ of the probability distribution $Y^{(i, j)}$ can be given as:

$$
H\left(\boldsymbol{r}^{(i, j)}\right)=-\sum_{l=1}^{M} \rho_{l}^{(i, j)} \log _{2} \rho_{l}^{(i, j)}
$$

Based on Algorithm 1, it is obvious to find that coefficient $M$ impacts the structure of the probability distribution $\gamma^{(i, j)}$. Thereby, the entropy $H\left(\Upsilon^{(i, j)}\right)$ will depend on the selection of coefficient $M$. Recall that the probability $\delta_{i}$ of uplink channels used by $\mathrm{SU} v_{i}$ determines the stability of successful transmission link $l_{(i, j)}$ or even the contact $\mathcal{C}^{(i, j)}$ between $\mathrm{SU} v_{i}$ and $\mathrm{SU}$ $v_{j}$ due to the impact of PUs' activities on the licensed uplink channels. As such, we formally devise a contact affinity metric to describe the stability of the contact between a pair of SUs. Without losing generality, the contact affinity metric $A_{(i, j)}$ between SU $v_{i}$ and $\mathrm{SU} v_{j}$ within time interval $\left[t_{0}, T\right]$ is formally expressed as:

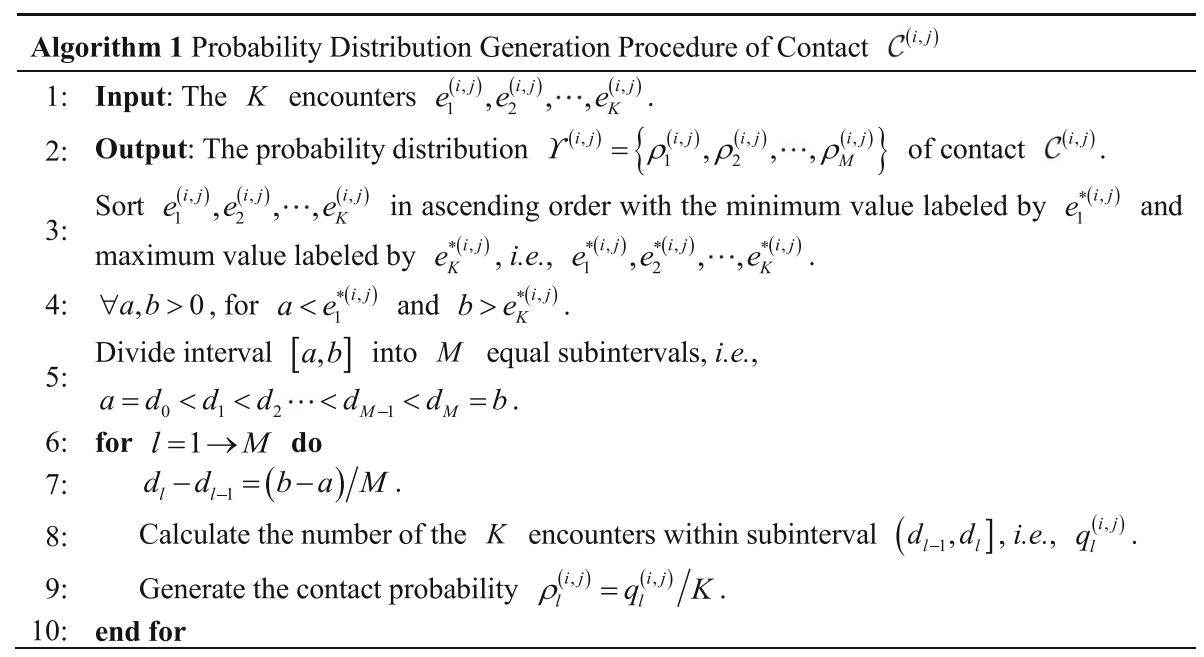


Table 1 Constrained relationship from algorithm 1

\begin{tabular}{lll}
\hline Subinterval & Number of encounters & Contact probability \\
\hline$\left(d_{0}, d_{1}\right]$ & $q_{1}^{(i, j)}$ & $\rho_{1}^{(i, j)}=q_{1}^{(i, j)} / K$ \\
$\left(d_{1}, d_{2}\right]$ & $q_{2}^{(i, j)}$ & $\rho_{2}^{(i, j)}=q_{2}^{(i, j)} / K$ \\
$\vdots$ & $\vdots$ & $\vdots$ \\
$\left(d_{M-1}, d_{M}\right]$ & $q_{M}^{(i, j)}$ & $\rho_{M}^{(i, j)}=q_{M}^{(i, j)} / K$ \\
\hline
\end{tabular}

$$
A_{(i, j)}=H\left(\gamma^{(i, j)}\right) \times \delta_{i} \times \delta_{j}
$$

\section{Problem formulation}

In this section, we intend to employ the differential game theoretic approach to formulate the PLPC problem in the physical layer and the HHCC problem in the transport layer. Clearly, the distributed strategy needs to be used to design the cross-layer optimization framework for congestion and power control due to the lack of centralized control and global information under an underlay CRANET scenario. As depicted in Fig. 3, each upstream SU will serve as power and rate controller in charge of joint optimized allocation of transmit power in the physical layer and data rate in the transport layer. Note that the change of power and rate will be continuous in time due to the fact that dynamic congestion and power control will be more realistic in a practical environment.

\subsection{Per-link power control in the physical layer}

Given the channel conditions, the capacity of successful transmission link between a pair of SUs is a nonconvex function of transmit power vector $\mathbf{P}$. In fact, increasing the link capacity on one link may reduce the link capacities on other links because of the mutual interference caused by SUs [20]. Instead, each SU is expected to increase its transmit power to provide as much link capacity that per-link flow requires [21]. However, this adjustment of power will generate extra interference to other SUs. It is necessary to achieve an optimal per-link power allocation in the physical layer for upstream SUs to meet link capacity supply for all the flows. By letting the transmit power of upstream SU $v_{i}$ equals the maximum transmit power threshold, we can easily obtain the maximum transmit power vector $\overline{\mathbf{P}}=\left\{\bar{p}_{1}, \bar{p}_{2}, \cdots, \bar{p}_{N}\right\}$. The transmission loss along link $l_{(i, b)}$ from upstream $\mathrm{SU} v_{i}$ to bottleneck SU $v_{b}$ on channel $c h_{\xi}$ is denoted by $\eta_{(i, b)}$. Due to the line-of-sight wireless transmission environment with slow-fading channel model, the transmission loss along link $l_{(i, b)}$ is represented by $\eta_{(i, b)}=\left[c /\left(4 \pi f_{\xi} \cdot\left\|\vartheta_{i}(t)-\vartheta_{b}(t)\right\|\right)\right]^{2}$, where $f_{\xi}$ is the carrier frequency operating on channel $c h_{\xi}$ and $c$ is the speed of light. Therefore, the maximum transmit power threshold $\bar{p}_{i}$ of upstream SU $v_{i}$ along link $l_{(i, b)}$ from upstream SU $v_{i}$ to bottleneck $\mathrm{SU} v_{b}$ can be formulated as:

$$
\bar{p}_{i}=\frac{\mathrm{p}_{(i, b)}^{\mathrm{ref}}}{\eta_{(i, b)}},
$$

where $\mathrm{p}_{(i, b)}^{\mathrm{ref}}$ is the received reference power at bottleneck SU $v_{b}$ along link $l_{(i, b)}$. Given the maximum transmit power threshold $\bar{p}_{i}$, the capacity of link $l_{(i, b)}$ from upstream SU $v_{i}$ to bottleneck SU $v_{b}$ can be denoted by $C_{(i, b)}^{*}(\overline{\mathbf{P}})$. Owing to maximum transmit power threshold, the value of power reduction for upstream SU $v_{i}$ is equal to $\bar{p}_{i}-p_{i}(t)$. Recall that bits-per-Joule capacity usually serves as a metric to measure the energy

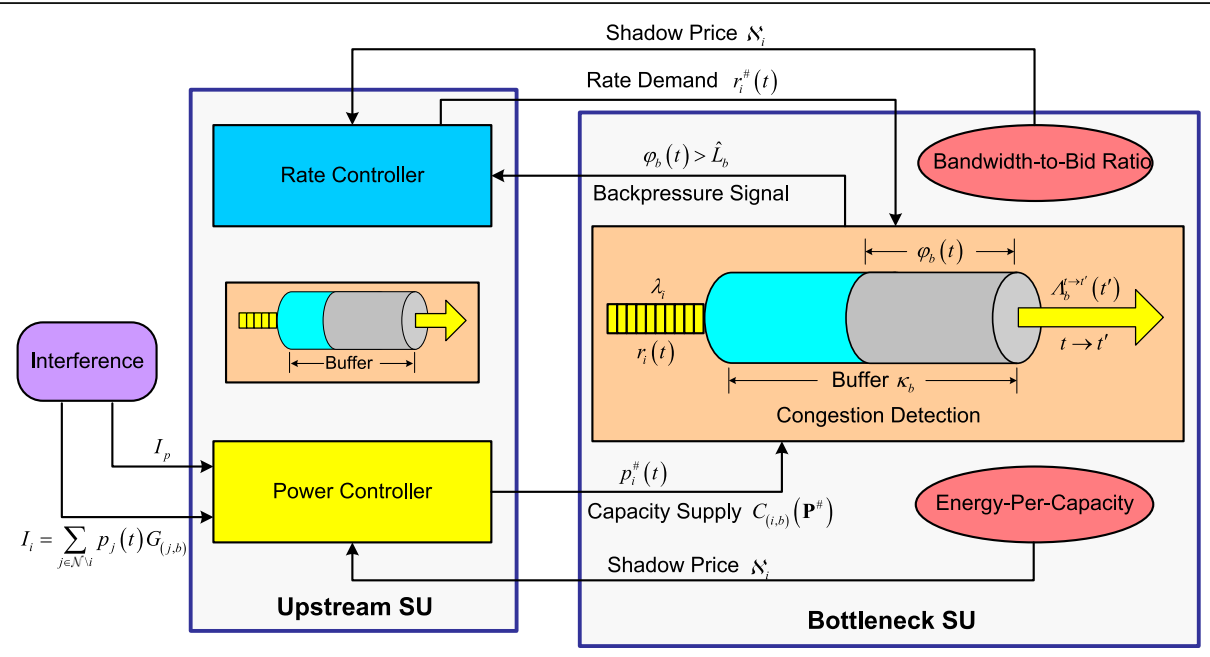

Fig. 3 Illustration of cross-layer optimization framework for hop-by-hop congestion control and per-link power control 
efficiency of a communication system [40]. Considering the impact of power reduction on energy efficiency with a link capacity constraint, energy efficiency for power reduction is formally defined as the power reduction achieved per capacity obtained under the maximum transmit power threshold. Thus, we plan to use the energy efficiency for power reduction to characterize a pricing factor of energy-per-capacity, aiming to design the revenue function of power reduction for upstream SU. Given the maximum transmit power vector $\overline{\mathbf{P}}$, the pricing factor $\Phi_{i}(t)$ of energy-percapacity of upstream SU $v_{i}$ at time $t$ can be formally defined as:

$$
\begin{gathered}
\Phi_{i}(t)=\frac{\bar{p}_{i}-p_{i}(t)}{C_{(i, b)}^{*}(\overline{\mathbf{P}})} \\
\frac{\frac{1}{T_{s}} \log _{2}\left(1+\chi \cdot \frac{\bar{p}_{i}-p_{i}(t)}{\sum_{j \in \mathcal{N} \backslash i} \bar{p}_{j} G_{(j, b)}+n_{b}+n_{0}}\right)}{}
\end{gathered}
$$

Revisiting the pricing factor of energy-per-capacity of upstream SU $v_{i}$, we define the revenue function of power reduction for upstream SU $v_{i}$ at time $t$ by attaining the product of the pricing factor together with power reduction value, i.e., $\Phi_{i}(t)\left(\bar{p}_{i}-p_{i}(t)\right)$. Let $\omega$ denote the pricing factor announced by upstream SU $v_{i}$ to measure the cost of the amount of aggregate power interference to PUs. The amount of aggregate power interference to PUs is denoted by $I(t)$. The cost function of aggregate power interference to PUs for upstream SU $v_{i}$ at time $t$ is given by $\omega I(t)$. Note that $I(t)$ is a dynamic variable influenced by transmit power $p_{i}(t)$ of upstream SU $v_{i}$ and instant level $I(t)$ within time interval $\left[t_{0}, T\right]$. Thus, the aggregate power interference $I(t)$ can be characterized as a linear differential equation given as:

$$
\left\{\begin{array}{l}
\frac{\mathrm{d} I(t)}{\mathrm{d} t}=\sum_{i \in \mathcal{N}} p_{i}(t)-\gamma I(t) \\
I\left(t_{0}\right)=I_{t_{0}}
\end{array}\right.
$$

where $\gamma>0$ is a penalty factor of the amount of aggregate power interference and $I_{t_{0}}$ is an initial aggregate power interference to PUs at time $t_{0}$. Therefore, based on both revenue and cost functions as mentioned, the utility function $U_{1}^{i}(t)$ of upstream $\mathrm{SU} v_{i}$ at time $t$ is given as:

$$
U_{1}^{i}(t)=\frac{\bar{p}_{i}-p_{i}(t)}{C_{(i, b)}^{*}(\overline{\mathbf{P}})}\left(\bar{p}_{i}-p_{i}(t)\right)-\omega I(t)
$$

Note that utility function $U_{1}^{i}(t)$ is a continuously differentiable function of $p_{i}(t)$ and $I(t)$. We can find that utility function in (14) mainly relies on pricing factor of energy-per-capacity in that the marginal effect on utility function stems from aggregate power interference. As shown in Fig. 3, we figuratively define pricing factor $\Phi_{i}(t)$ as a shadow price $\boldsymbol{\aleph}_{i}$ which is a function of transmit power of upstream SU $v_{i}$. Our optimization objective is to maximize utility function $U_{1}^{i}(t)$ by choosing optimal transmit power $p_{i}^{\#}(t)$ of upstream SU $v_{i}$ according to $\Phi_{i}(t)$ and $\omega$ :

$\underset{p_{i}(t)}{\operatorname{Maximize}}: \quad \int_{t_{0}}^{T}\left(\frac{\bar{p}_{i}-p_{i}(t)}{C_{(i, b)}^{*}(\overline{\mathbf{P}})}\left(\bar{p}_{i}-p_{i}(t)\right)-\omega I(t)\right) e^{-a\left(t-t_{0}\right)} \mathrm{d} t$

where $a$ is a discount factor, for $0<a<1$. Note that discount factor $a$ is an exponential factor between 0 and 1 by which the future utility must be multiplied in order to obtain the present value under the underlying structure of differential game. Hence, utility function $U_{1}^{i}(t)$ has to be discounted by the factor $e^{-a\left(t-t_{0}\right)}$. Formally, the PLPC problem in the physical layer is formulated as a differential game model $\Gamma_{P L P C}$ :

$$
\Gamma_{P L P C}=\left\{\mathcal{N},\left\{p_{i}(t)\right\}_{i \in \mathcal{N}}, I(t),\left\{U_{1}^{i}(t)\right\}_{i \in \mathcal{N}}\right\}
$$

where

- Player set $\mathcal{N}: \mathcal{N}=\{1,2, \cdots, N\}$ is the set of all the upstream SUs in the PLPC problem as power controllers playing the game. Note that upstream $\mathrm{SU} v_{i}$ stands for the $i$ th player which is a rational policy maker and acts throughout time interval $\left[t_{0}, T\right]$.

- Set of strategies $\left\{p_{i}(t)\right\}_{i \in \mathcal{N}}$ : The strategy of the $i$ th player refers to its instant transmit power limited by the maximum transmit power threshold, i.e., $p_{i}(t) \in\left[0, \bar{p}_{i}\right)$.

- State variable $I(t)$ : The state variable of the $i$ th player corresponds to the amount of aggregate power interference to PUs.

- Set of utility functions $\left\{U_{1}^{i}(t)\right\}_{i \in \mathcal{N}}: U_{1}^{i}(t)$ is the utility function of the $i$ th player. The objective of the $i$ th player is to maximize its utility function by rationally selecting optimal strategy $p_{i}^{\#}(t)$ and optimal state $I^{\#}(t)$.

3.2 Hop-by-hop congestion control in the transport layer Under the scenario that multiple flows from upstream SU2, SU6, and SU8 via single hop converge at bottleneck SU3 in Fig. 2, bottleneck SU3 is a little more inclined to be a congested SU when offered data load exceeds available capacity of SU3 
due to buffer overflow. The amount of data packets with elastic traffic in the buffer of bottleneck SU $v_{b}$ at time $t$ is denoted by $\phi_{b}(t)$. Given time $t, t^{\prime} \in\left[t_{0}\right.$, $T]$ and $t<t$, the amount of data packets $\phi_{b}(t)$ of bottleneck SU $v_{b}$ within the time interval $\left[t, t^{\prime}\right]$ satisfies the following iterative equation:

$\phi_{b}\left(t^{\prime}\right)=\max \left\{\min \left\{\phi_{b}(t)+\phi_{b}^{t \rightarrow t^{\prime}}\left(t^{\prime}\right), \kappa_{b}\right\}-\Lambda_{b}^{t \rightarrow t^{\prime}}\left(t^{\prime}\right), 0\right\}$,

where $\kappa_{b}$ is the buffer size of bottleneck SU $v_{b}$, and $\phi_{b}^{t \rightarrow t^{\prime}}\left(t^{\prime}\right)$ and $\Lambda_{b}^{t \rightarrow t^{\prime}}\left(t^{\prime}\right)$ are the amount of data packets accumulated in the buffer of bottleneck SU $v_{b}$ and the amount of data packets that could be delivered successfully to downstream SUs, respectively. Considering the constraint of the saturation value $\hat{L}_{b}$ of the buffer of bottleneck SU $v_{b}$, we have the buffer constraint $\phi_{b}(t) \leq \hat{L}_{b}$ to guarantee that bottleneck SU $v_{b}$ will not become the real congested SU.

In the end-to-end congestion control, the congestion detection information is piggybacked over data packets to the destination and then sent to the source through the acknowledgement packet from the destination. However, to feed the congestion detection information back to the upstream SU2, SU6, and SU8, bottleneck SU3 will generate a backpressure signal to notify that the congestion occurs as shown in Fig. 2. Compared with the end-to-end mechanism, the backpressure signal is directly sent back to the corresponding upstream SUs from the bottleneck SU3 via single hop. Instead of passing the congestion detection information sent to the source in an end-to-end approach, the idea of hopby-hop congestion control policy in this paper is that upstream SU $v_{i}$ directly adjusts the data rate $r_{i}(t)$ according to the backpressure signal of bottleneck SU $v_{b}$ when $\phi_{b}(t)>\hat{L}_{b}$. Based on the radio transceiver equipped by each $\mathrm{SU}$, the backpressure signal is assumed to be transmitted through a common control channel.

Given the received SINR of link $l_{(i, b)}$, we proceed to derive the required bandwidth of upstream SU $v_{i}$ for transmissions of data packets with elastic traffic. According to Shannon's capacity formula, the required bandwidth of upstream $\mathrm{SU} v_{i}$ can be expressed by $r_{i}(t) / \log _{2}\left(1+X \cdot \operatorname{SINR}_{(i, b)}(\mathbf{P})\right)$. We further assume that bottleneck $\mathrm{SU} v_{b}$ acts as a bidder and pays for upstream SU $v_{i}$ to accommodate the consumption of its network resources while regulating the data rate $r_{i}(t)$. We use $x(t)$ to represent the congestion bid that bottleneck $\mathrm{SU} v_{b}$ is willing to pay. We then characterize a bandwidth-to-bid ratio aiming to describe the efficiency of bid with regard to the required bandwidth, i.e., $\left[r_{i}(t) / \log _{2}(1+\chi\right.$. $\left.\left.\operatorname{SINR}_{(i, b)}(\mathbf{P})\right)\right] / x(t)$. Hence, the cost function $C_{i}(t)$ of upstream SU $v_{i}$ at time $t$ can be defined as:

$$
C_{i}(t)=r_{i}(t) \times \frac{r_{i}(t) / \log _{2}\left(1+\chi \cdot \operatorname{SINR}_{(i, b)}(\mathbf{P})\right)}{x(t)} \times D_{i} .
$$

We also use the bandwidth-to-bid ratio to figuratively express the shadow price $\boldsymbol{\aleph}_{i}$ which is a function of the data rate of upstream SU $v_{i}$. Note that the shadow price $\boldsymbol{\aleph}_{i}$ depends on an optimal per-link power allocation in the PLPC problem due to the constraint of the received SINR of link $l_{(i, b)}$ in bandwidth-to-bid ratio. In this way, we turn our attention to the cross-layer coordination mechanism between capacity supply by power controller and rate demand by rate controller based on shadow prices $\boldsymbol{N}_{i}$ and $\boldsymbol{\kappa}_{i}$ as shown in Fig. 3. By taking into account the stability of the contact between a pair of SUs, we conclude that $A_{(i \text {, }}$ b) $x(t)$ is the accumulated revenue obtained by upstream $\mathrm{SU} v_{i}$ that bottleneck $\mathrm{SU} v_{b}$ needs to pay. We also remark that the congestion bid $x(t)$ is a dynamic variable influenced by rate $r_{i}(t)$ as well as by instant level of $x(t)$ within time interval $\left[t_{0}, T\right]$. Accordingly, the congestion bid $x(t)$ can be formulated as a linear differential equation:

$$
\left\{\begin{array}{l}
\frac{\mathrm{d} x(t)}{\mathrm{d} t}=x(t)-\sum_{i \in \mathcal{N}} v r_{i}(t) \\
x\left(t_{0}\right)=x_{t_{0}}
\end{array}\right.
$$

where $x_{t_{0}}$ is an initial congestion bid that bottleneck SU $v_{b}$ needs to pay at time $t_{0}$ and $v$ is an average bid per rate, which is assumed to be a unit value for all the upstream SUs, i.e., $v=1$. Formally, based on both revenue and cost functions as stated before, the utility function $U_{2}^{i}(t)$ of upstream SU $v_{i}$ at time $t$ can be expressed as:

$$
U_{2}^{i}(t)=A_{(i, b)} x(t)-C_{i}(t) .
$$

Noticing that utility function $U_{2}^{i}(t)$ is also a continuously differentiable function of $r_{i}(t)$ and $x(t)$. Our optimization objective is to maximize utility function $U_{2}^{i}(t)$ by choosing optimal data rate $r_{i}^{\#}(t)$ of upstream SU $v_{i}$ while satisfying the buffer constraint at the same time:

$$
\underset{r_{i}(t)}{\operatorname{Maximize}:} \quad \int_{t_{0}}^{T}\left(A_{(i, b)} x(t)-C_{i}(t)\right) e^{-\tau\left(t-t_{0}\right)} \mathrm{d} t
$$

where $\tau$ is a discount factor, for $0<\tau<1$. Similarly, $U_{2}^{i}(t)$ will also be discounted by the factor $e^{-\tau\left(t-t_{0}\right)}$. Correspondingly, the HHCC problem in the transport layer can be also defined as a differential game model $\Gamma_{H H C C}$ : 


$$
\Gamma_{H H C C}=\left\{\mathcal{N},\left\{r_{i}(t)\right\}_{i \in \mathcal{N}}, x(t),\left\{U_{2}^{i}(t)\right\}_{i \in \mathcal{N}}\right\}
$$

where

- Player set $\mathcal{N}: \mathcal{N}=\{1,2, \cdots, N\}$ is the set of all the upstream SUs in the HHCC problem as rate controllers playing the game. Upstream $\mathrm{SU} v_{i}$ is also known as the $i$ th player which is a rational policy maker and act throughout time interval $\left[t_{0}, T\right]$.

- Set of strategies $\left\{r_{i}(t)\right\}_{i \in \mathcal{N}}$ : The strategy of the $i$ th player corresponds to its instant data rate $r_{i}(t)$.

- State variable $x(t)$ : The state variable of the $i$ th player refers to the congestion bid that bottleneck $\mathrm{SU} v_{b}$ is willing to pay.

- Set of utility functions $\left\{U_{2}^{i}(t)\right\}_{i \in \mathcal{N}}: U_{2}^{i}(t)$ is the utility function of the $i$ th player. The objective of the $i$ th player is to maximize its utility function by rationally choosing optimal strategy $r_{i}^{\#}(t)$ and optimal state $x^{\#}(t)$.

\section{Optimal solution and distributed implementation}

Conventionally, upstream SUs as players of the game are expected to act cooperatively and maximize their joint utility functions with fairness for players by constituting the collaborative coalition. As a result, the global optimization of transmit power and data rate will be attained through cooperation among players with group rationality, which has been recently reported in a cooperative bargaining game [41]. However, each upstream SU is unwilling to jointly adjust the power and rate because of the selfish behavior in forwarding data packets. This is a natural idea due to the fact that the transmissions lead to the consumption of network resources of upstream SUs, such as energy and spectrum. Therefore, the cross-layer optimization framework for congestion and power control will be restricted to noncooperation scenario. In the noncooperative differential game models $\Gamma_{P L P C}$ and $\Gamma_{H H C C}$, the $i$ th player competes to maximize the present value of its utility function derived over time interval $\left[t_{0}, T\right]$. For mathematical tractability, we define the starting time of the differential game models $\Gamma_{P L P C}$ and $\Gamma_{H H C C}$ as $t_{0}=0$ hereinafter, but the results can be easily extended to more general cases.

\subsection{Optimal solution to $\Gamma_{P L P C}$}

For the noncooperation scenario, we formulate a dynamic optimization problem $\mathbb{P} 1$ to derive the optimal solution to the noncooperative differential game model $\Gamma_{P L P C}$ by taking into account the utility function maximization problem coupled with the linear differential equation constraint in (13):

$$
\begin{gathered}
\mathbb{P} 1 \quad \underset{p_{i}(t)}{\text { Maximize }}: \int_{0}^{T}\left(\frac{\bar{p}_{i}-p_{i}(t)}{C_{(i, b)}^{*}(\overline{\mathbf{P}})}\left(\bar{p}_{i}-p_{i}(t)\right)-\omega I(t)\right) e^{-a t} \mathrm{~d} t \\
\text { Subject to }: \frac{\mathrm{d} I(t)}{\mathrm{d} t}=\sum_{i \in \mathcal{N}} p_{i}(t)-\gamma I(t), \\
I\left(t_{0}=0\right)=I_{0} .
\end{gathered}
$$

We aim at deriving an optimal solution to $\mathbb{P} 1$ by employing the theory of dynamic programming developed by Bellman [42]. Remark that the optimal solution is also viewed as a Nash equilibrium solution to $\mathbb{P} 1$ if all the players play noncooperatively. Here, we relax the terminal time of $\Gamma_{P L P C}$ to explore when $T$ approaches $\infty$ (i.e., $T \rightarrow \infty$ ) as an infinite time horizon. It is more realistic to obtain the long-term optimal power allocation for upstream SUs due to spectrum underlay strategy with cellular primary network. We use $p_{i}^{\#}(t)$ to represent the optimal solution to $\mathbb{P} 1$ and assume that there exists a continuously differentiable function $V^{i}\left(p_{i}, I\right)$ satisfying the following partial differential equation:

$$
a V^{i}\left(p_{i}, I\right)=\underset{p_{i}(t)}{\operatorname{Maximize}}:\left\{\frac{\left(\bar{p}_{i}-p_{i}(t)\right)^{2}}{C_{(i, b)}^{*}(\mathbf{P})}-\omega I(t)+\frac{\partial V^{i}\left(p_{i}, I\right)}{\partial I}\left(\sum_{j \in M i} p_{j}^{\#}(t)+p_{i}(t)-\gamma I(t)\right)\right\} .
$$

4.1.0.1 Theorem 1 A vector of optimal transmit power $\mathbf{P}^{\#}$ $=\left\{p_{1}^{\#}(t), p_{2}^{\#}(t), \cdots, p_{N}^{\#}(t)\right\}$ of upstream SUs constitutes a Nash equilibrium solution to $\mathbb{P} 1$ if and only if the optimal transmit power $p_{i}^{\#}(t)$ of the ith player and the continuously differentiable function $V^{i}\left(p_{i}, I\right)$ can be formulated as follows:

$$
\begin{aligned}
p_{i}^{\#}(t) & =\bar{p}_{i}-\frac{\omega C_{(i, b)}^{*}(\overline{\mathbf{P}})}{2(a+\gamma)}, \\
V^{i}\left(p_{i}^{\#}, I\right) & =\frac{\omega}{a(a+\gamma)}\left(\frac{\omega C_{(i, b)}^{*}(\overline{\mathbf{P}})(1+2 N)}{4(a+\gamma)}-a I-\sum_{i \in \mathcal{N}} \bar{p}_{i}\right) .
\end{aligned}
$$

Proof: See Appendix 1. $\mathbf{m .}$

From Theorem 1, we can observe that the existence and uniqueness of the Nash equilibrium point to $\mathbb{P} 1$ are guaranteed under the constraint of analytical solution in (25) and (26). It is also revealed that the optimal transmit power $p_{i}^{\#}(t)$ has been characterized by a fixed and unique value in (25). Evidently, Theorem 1 mathematically ensures the convergence of $p_{i}^{\#}(t)$ to a Nash equilibrium point. The key point to derive the optimal solution to the differential game model $\Gamma_{P L P C}$ is illustrated with a block diagram shown in Fig. 4a.

4.1.0.2 Proposition 1 For the given large-scale slowfading channel model, by letting $G_{1}=\varpi_{\mathrm{PBS}} /\left(10^{6} g_{0}\right)^{N}$, the 


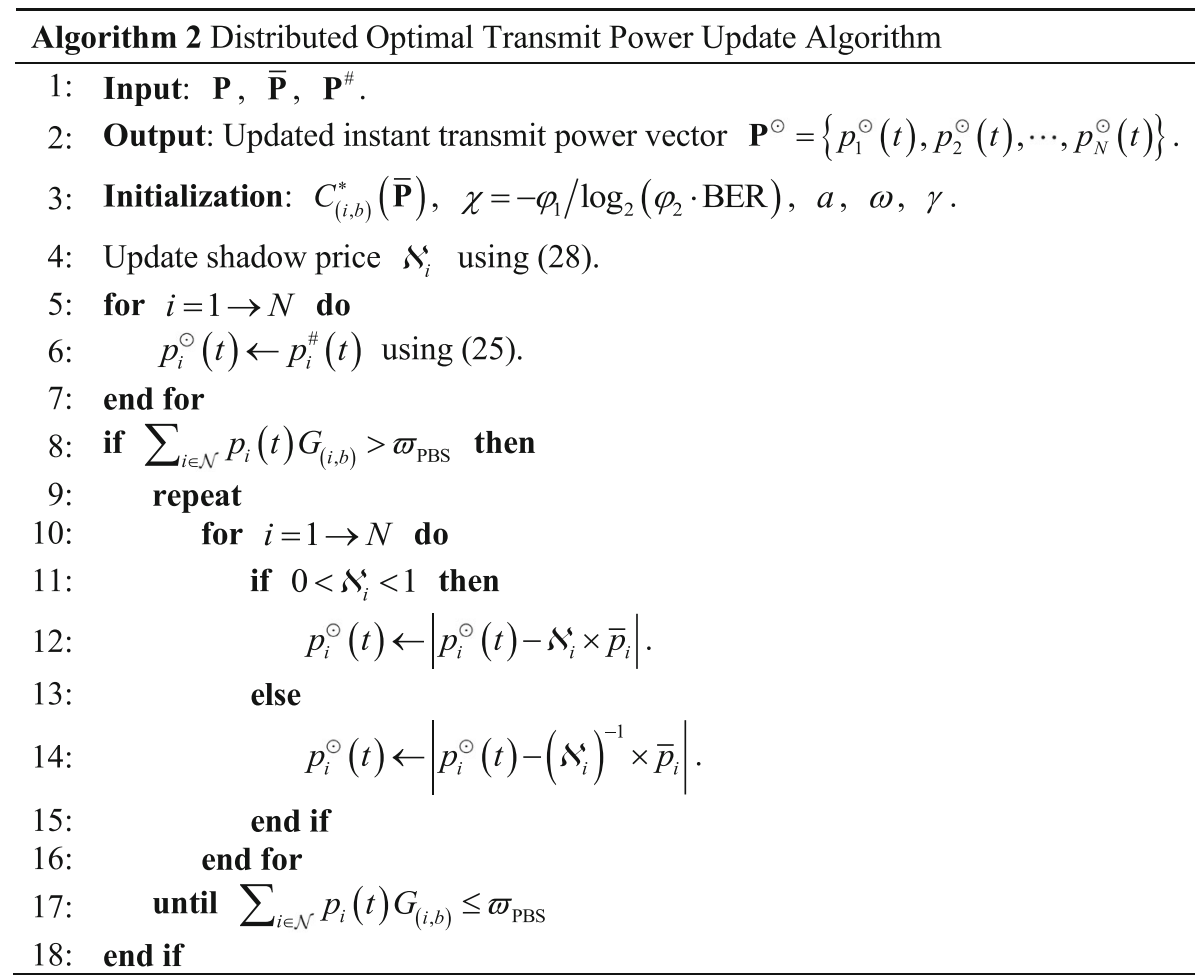

optimal transmit power $p_{i}^{\#}(t)$ of the ith player should follow the interference power constraint:

$$
\prod_{i \in \mathcal{N}} p_{i}^{\#}(t) \leq G_{1} \prod_{i \in \mathcal{N}}\left\|\vartheta_{i}(t)-\vartheta_{b}(t)\right\|^{4}
$$

\section{Proof: See Appendix 2.}

Note that the optimal transmit power $p_{i}^{\#}(t)$ of the $i$ th player is fully constrained by the Euclidean distance between upstream SU $v_{i}$ and bottleneck SU $v_{b}$ under the given channel model. Substituting for $p_{i}^{\#}(t)$ with its expression from Theorem 1 and taking into account the previous expression of shadow price $\boldsymbol{\aleph}_{i}$, we can easily rewrite $\boldsymbol{\aleph}_{i}$ as:

$$
\boldsymbol{\aleph}_{i}=\frac{\omega}{2(a+\gamma)}
$$

Apparently, shadow price $\boldsymbol{\aleph}_{i}$ tends to be a constant value for all the upstream SUs. Although (25) and (26) offer an analytical solution to $\mathbb{P} 1$, it still remains to design an algorithm to ensure fast convergence of the update of optimal transmit power. Therefore, we devise a distributed optimal transmit power update (OTPU) strategy given in Algorithm 2 to update the optimal transmit power vector $\mathbf{P}^{\#}$ for upstream SUs. Similar to [43], shadow price $\boldsymbol{N}_{i}$ in Algorithm 2 needs to carefully be chosen to ensure fast convergence of the update of instant transmit power $p_{i}^{\odot}(t)$. It is also noted that the update of instant transmit power $p_{i}^{\odot}(t)$ for upstream SU $v_{i}$ can be made locally according to its optimal transmit power $p_{i}^{\#}(t)$ along with interference power constraint.

\subsection{Optimal solution to $\Gamma_{H H C C}$}

For notational simplicity, we begin by defining a notation $B_{(i, b)} \triangleq-D_{i} / \log _{2}\left(1+\chi \cdot \operatorname{SINR}_{(i, b)}(\mathbf{P})\right)$. For the noncooperation scenario, we formulate a dynamic optimization problem $\mathbb{P} 2$ to derive the optimal solution to the noncooperative differential game model $\Gamma_{H H C C}$ by taking into account both the utility function maximization problem and the linear differential equation constraint in (19):

$$
\begin{gathered}
\mathbb{P} 2 \underset{r_{i}(t)}{\operatorname{Maximize}}: \int_{0}^{T}\left(A_{(i, b)} x(t)+B_{(i, b)} \frac{r_{i}^{2}(t)}{x(t)}\right) e^{-\tau t} \mathrm{~d} t \\
\text { Subject to }: \frac{\mathrm{d} x(t)}{\mathrm{d} t}=x(t)-\sum_{i \in \mathcal{N}} r_{i}(t), \\
x\left(t_{0}=0\right)=x_{0} .
\end{gathered}
$$

We turn to take advantage of the theory of maximum principle developed by Pontryagin [42] to derive an optimal solution or a Nash equilibrium solution to $\mathbb{P} 2$. We further use $r_{i}^{\#}(t)$ to represent the optimal solution to $\mathbb{P} 2$ and assume that there exists a continuously differentiable function $W^{i}\left(r_{i}, x\right)$ satisfying the partial differential equation as follows: 


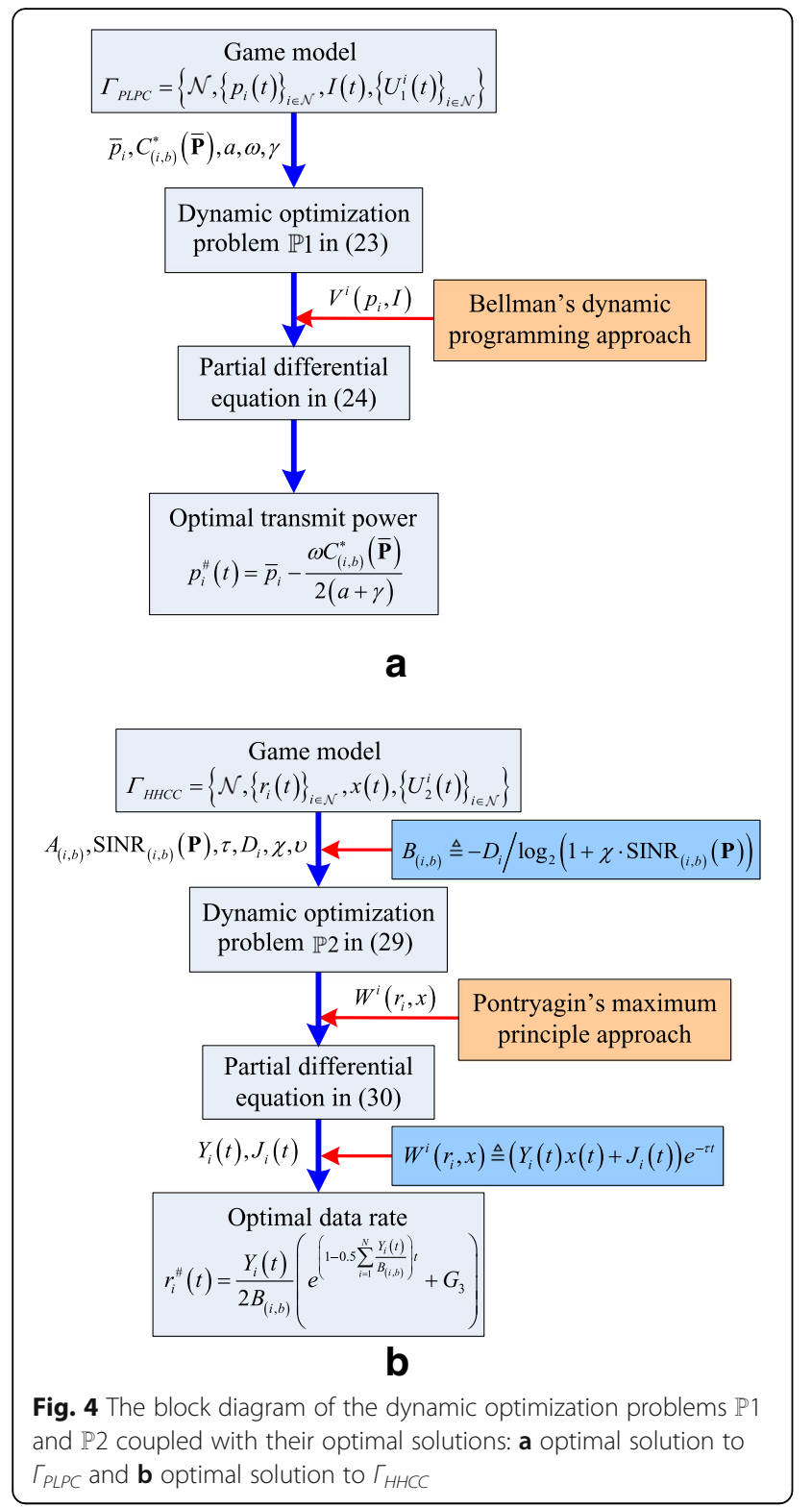

$$
\begin{aligned}
-\frac{\partial W^{i}\left(r_{i}, x\right)}{\partial s}= & \underset{r_{i}(t)}{\operatorname{Maximize}}:\left\{\left(A_{(i, b)} x(t)+B_{(i, b)} \frac{r_{i}^{2}(t)}{x(t)}\right) e^{-t t}\right. \\
& \left.+\frac{\partial W^{i}\left(r_{i}, x\right)}{\partial x}\left(x(t)-\sum_{j \in \mathcal{N} \backslash i} r_{j}^{\#}(t)-r_{i}(t)\right)\right\} .
\end{aligned}
$$

For tractability, we introduce two extra introduced auxiliary variables $Y_{i}(t)$ and $J_{i}(t)$ to characterize $W^{i}\left(r_{i}, x\right)$. Specifically, we define $W^{i}\left(r_{i}, x\right) \triangleq\left(Y_{i}(t) x(t)+J_{i}(t)\right) e^{-t t}$.

4.2.0.1 Theorem 2 A vector of optimal data rate $\mathbf{R}^{\#}=\{$ $\left.r_{1}^{\#}(t), r_{2}^{\#}(t), \cdots, r_{N}^{\#}(t)\right\}$ of upstream SUs constitutes a Nash equilibrium solution to $\mathbb{P} 2$ if and only if the optimal data rate $r_{i}^{\#}(t)$ of the ith player can be expressed as:

$$
r_{i}^{\#}(t)=\frac{Y_{i}(t) x(t)}{2 B_{(i, b)}},
$$

where $Y_{i}(t)$ and $J_{i}(t)$ satisfy the following differential equations:

$$
\begin{aligned}
& \mathrm{d} Y_{i} \frac{(t)}{\mathrm{d} t}=\frac{1}{4 B_{(i, b)}} Y_{i}^{2}(t)+(\tau-1) Y_{i}(t)-A_{(i, b)}+\frac{Y_{i}(t)}{2} \sum_{j \in \mathcal{N} \backslash i} \frac{Y_{j}(t)}{B_{(j, b)}}, \\
& \frac{\mathrm{d} J_{i}(t)}{\mathrm{d} t}=\tau \cdot J_{i}(t) .
\end{aligned}
$$

Proof: See Appendix 3..

For notational simplicity, we set $\Omega_{(i, b)}=1 /\left(4 B_{(i, b)}\right)+0$. $5 \sum_{j \in \mathcal{N} \backslash i} 1 / B_{(j, b)}$ and $\varepsilon=\sqrt{(\tau-1)^{2}+4 \Omega_{(i, b)} A_{(i, b)}}$, for $4 \Omega_{(i,}$ ${ }_{b)} A_{(i, b)}+(\tau-1)^{2}>0$. We also denote $G_{2}$ as a constant number. Substituting $\Omega_{(i, b)}$ into $\varepsilon$, we can rewrite $\varepsilon$ as follows:

$$
\varepsilon=\sqrt{(\tau-1)^{2}+\frac{A_{(i, b)}}{B_{(i, b)}}+2 A_{(i, b)} \sum_{j \in \mathcal{N} \backslash i} \frac{1}{B_{(j, b)}}} .
$$

4.2.0.2 Proposition 2 The auxiliary variable $Y_{i}(t)$ in the Nash equilibrium solution $r_{i}^{\#}(t)$ to $\mathbb{P} 2$ can be further given as:

$$
Y_{i}(t)=\frac{((\tau-1)+\varepsilon) e^{\left(t-G_{2}\right) \varepsilon}+\varepsilon-\tau+1}{2 \Omega_{(i, b)}\left(1-e^{\left(t-G_{2}\right) \varepsilon}\right)} .
$$

Proof: See Appendix 4. $\mathbf{m}$.

Combining $Y_{i}(t)$ in (35) and $\varepsilon$ in (34) yields the expression for $Y_{i}(t)$ as:

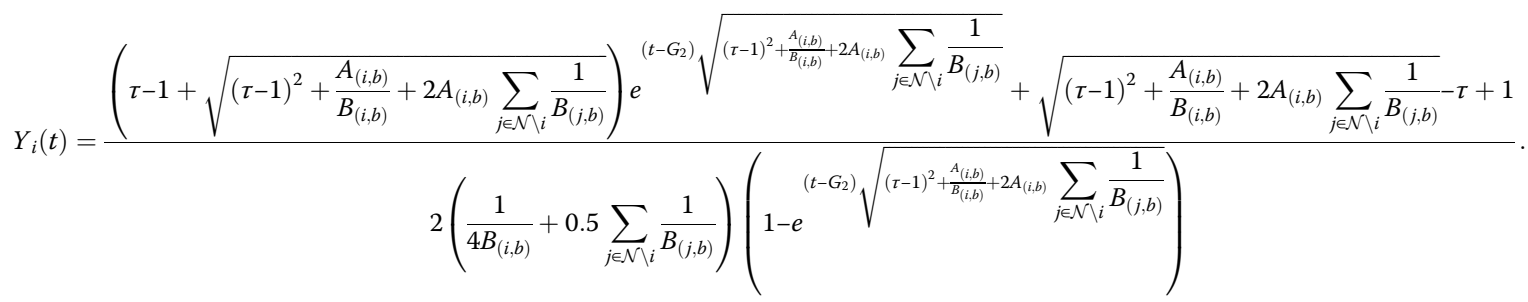


Substituting $B_{(i, b)} \triangleq-D_{i} / \log _{2}\left(1+\chi \cdot \operatorname{SINR}_{(i, b)}(\mathbf{P})\right)$ into (36), we can rewrite $Y_{i}(t)$ as follows:

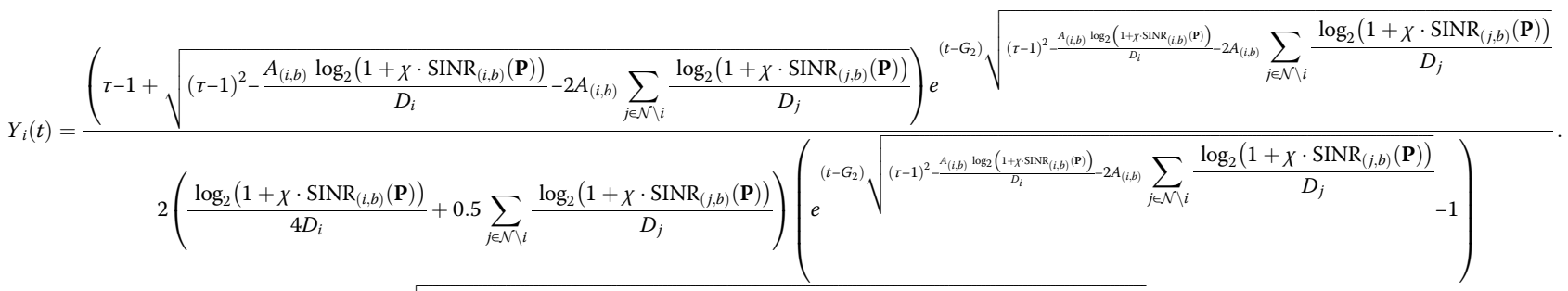

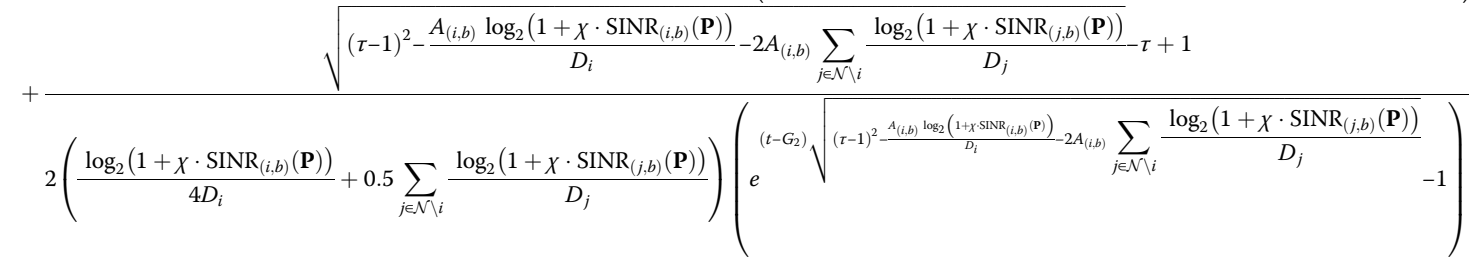

Let $G_{3}$ be a constant number. Based on (31) and (29), the optimal data rate $r_{i}^{\#}(t)$ and the optimal state variable $x^{\#}(t)$ associated with $r_{i}^{\#}(t)$ can be described as:

$$
\begin{aligned}
& r_{i}^{\#}(t)=\frac{Y_{i}(t)}{2 B_{(i, b)}}\left(e^{\left(1-0.5 \sum_{i=1}^{N} \frac{Y_{i}(t)}{B(i, b)}\right) t}+G_{3}\right), \\
& x^{\#}(t)=e^{\left(1-0.5 \sum_{i=1}^{N} \frac{Y_{i}(t)}{B(i, b)}\right) t}+G_{3} .
\end{aligned}
$$

From (38), we can see that the optimal data rate $r_{i}^{\#}(t)$ is determined by both $B_{(i, b)}$ and auxiliary variable $Y_{i}(t)$ under the received $\operatorname{SINR} \operatorname{SINR}_{(i, b)}(\mathbf{P})$ of link $l_{(i, b)}$. Unfortunately, it is difficult to directly obtain the relationship between $\operatorname{SINR}_{(i, b)}(\mathbf{P})$ and $r_{i}^{\#}(t)$ through an analytical derivation because $Y_{i}(t)$ cannot be further simplified into a concise structure. Thereby, given the channel gain $G_{(i, b)}$ of link $l_{(i, b)}$ under the large-scale slow-fading channel model, we use the numerical simulations to validate the effectiveness of the optimal data rate $r_{i}^{\#}(t)$ of upstream SU $v_{i}$. We further remark that Theorem 2 and Proposition 2 characterize the existence of the Nash equilibrium point to $\mathbb{P} 2$. It should be also admitted that the optimal data rate $r_{i}^{\#}(t)$ has been formulated as a fixed and unique value in (38) by using auxiliary variable $Y_{i}(t)$ in (37). Correspondingly, Theorem 2 mathematically ensures the convergence of $r_{i}^{\#}(t)$ to a Nash equilibrium point. The key point to derive the optimal solution to the differential game model $\Gamma_{H H C C}$ is also illustrated with a block diagram depicted in Fig. $4 \mathrm{~b}$. With the help of vectors $\mathbf{R}^{\#}$ and $\mathbf{P}^{\#}$, shadow price $\boldsymbol{\kappa}_{i}$ can be calculated as:

$$
\boldsymbol{\aleph}_{i}=\left(\frac{1}{\log _{2}\left(1+\chi \cdot \operatorname{SINR}_{(i, b)}\left(\mathbf{P}^{\#}\right)\right)}\right) \frac{r_{i}^{\#}(t)}{x^{\#}(t)}
$$

4.2.0.3 Proposition 3 For the given vector $\mathbf{R}^{\#}$ and vector $\mathbf{P}^{\#}$, a strict lower bound of shadow price $\boldsymbol{N}_{i}$ can be approximately calculated as follows:

$$
\boldsymbol{\kappa}_{i} \geq\left(\log _{2}\left(x \cdot \frac{p_{i}^{\#}(t)}{\sum_{j \in \mathcal{N} \backslash i} p_{j}^{\#}(t)}\right)\right)^{-1} \frac{r_{i}^{\#}(t)}{x^{\#}(t)}
$$

\section{Proof: See Appendix 5.}

Let $\mathrm{r}_{(i, b)}^{\text {base }}$ represent the baseline rate along link $l_{(i, b)}$ from upstream SU $v_{i}$ to bottleneck SU $v_{b}$. To guarantee the constraint of the saturation value of the buffer of bottleneck SU $v_{b}$, we design a distributed algorithm to obtain the optimal data rate update (ODRU) for upstream SUs as summarized in Algorithm 3, where $\mathbf{R}$ $=\left\{r_{1}(t), r_{2}(t), \cdots, r_{N}(t)\right\}$ denotes the data rate vector of $N$ upstream SUs at time $t$. A simple yet effective way to locally adjust the instant data rate $r_{i}^{\odot}(t)$ of upstream SU $v_{i}$ is to employ the optimal data rate $r_{i}^{\#}(t)$ under the condition of buffer constraint $\phi_{b}(t) \leq \hat{L}_{b}$. Also, baseline rate $\mathrm{r}_{(i, b)}^{\text {base }}$ in Algorithm 3 should be carefully chosen to ensure the effectiveness of instant data rate. 


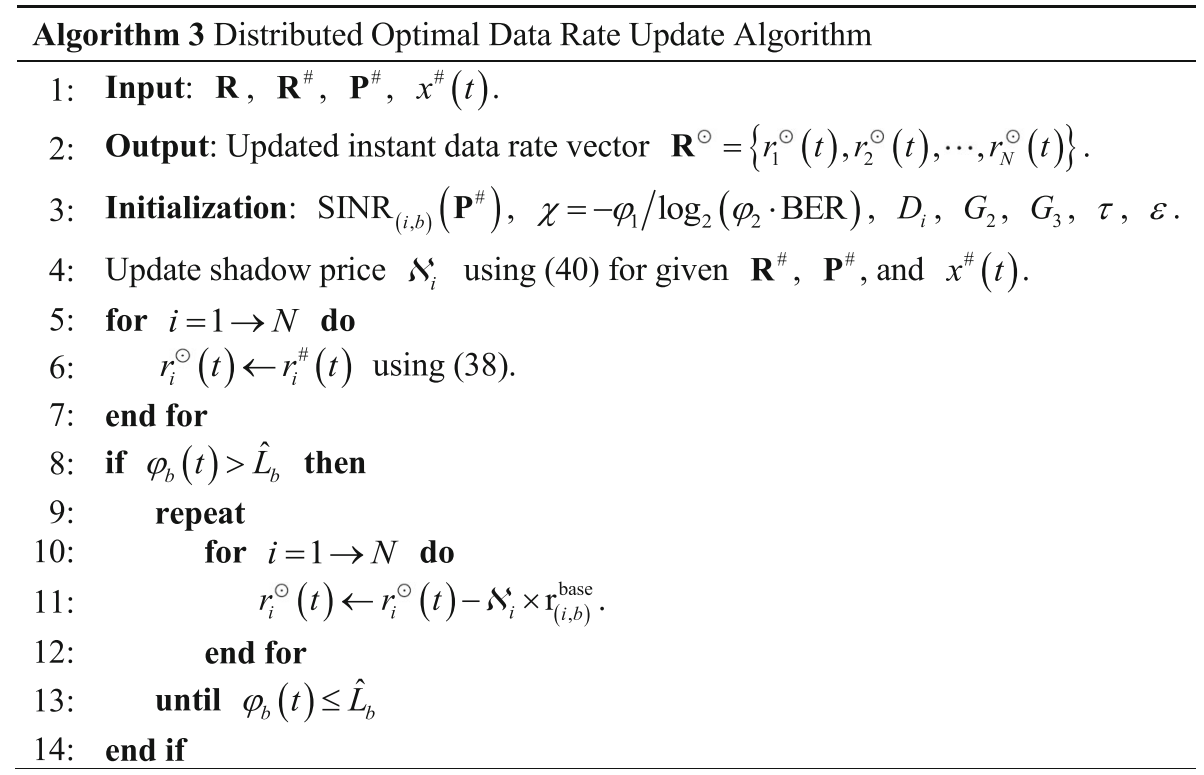

\subsection{Distributed implementation}

So far, we have devised Algorithms 2 and 3 to satisfy the interference power constraint along with buffer constraint by locally adjusting the optimal transmit power and the optimal data rate, respectively. In what follows, we would like to describe the distributed implementation strategy to realize the cross-layer optimization framework for congestion and power control by jointly optimizing PLPCHHCC simultaneously. In conclusion, the crosslayer optimization scheme for joint PLPC-HHCC design is implemented in a distributed manner as follows:

\subsubsection{Shadow price}

Update shadow price $\boldsymbol{\aleph}_{i}$ using (28) and shadow price $\boldsymbol{\aleph}_{i}$ using (40), respectively.

\subsubsection{Power controller in the physical layer}

For each upstream SU, we initially assign optimal transmit power $p_{i}^{\#}(t)$ using (25) to update instant transmit power $p_{i}^{\odot}(t)$ at power controller. Due to the interference power constraint in (6) to protect PUs, $p_{i}^{\odot}(t)$ should satisfy the following distributed powerupdate function when $\sum_{i \in \mathcal{N}} p_{i}(t) G_{(i, b)}>\varpi_{\mathrm{PBS}}$ via OTPU algorithm:

$$
p_{i}^{\odot}(t)= \begin{cases}\left|p_{i}^{\odot}(t)-\boldsymbol{\kappa}_{i} \times \bar{p}_{i}\right|, & \text { for } \quad 0<\boldsymbol{\kappa}_{i}<1 \\ \left|p_{i}^{\odot}(t)-\left(\boldsymbol{\aleph}_{i}\right)^{-1} \times \bar{p}_{i}\right|, & \text { for } \quad \boldsymbol{\aleph}_{i} \geq 1\end{cases}
$$

\subsubsection{Rate controller in the transport layer}

For each upstream SU, we also initially assign optimal data rate $r_{i}^{\#}(t)$ using (38) to update instant data rate $r_{i}^{\odot}($ $t)$ at rate controller. Owing to the buffer constraint $\phi_{b}(t$ )$\leq \hat{L}_{b}$ to guarantee that bottleneck SU $v_{b}$ will not become congested, $r_{i}^{\odot}(t)$ should be subject to the following distributed rate update function when $\phi_{b}(t)>\hat{L}_{b}$ via ODRU algorithm:

$$
r_{i}^{\odot}(t) \leftarrow r_{i}^{\odot}(t)-\boldsymbol{\aleph}_{i} \times \mathrm{r}_{(i, b)}^{\text {base }}
$$

\subsubsection{Cross-layer coordination mechanism}

With the aid of the updated power $p_{i}^{\odot}(t)$, the link capacity supply $C_{(i, b)}\left(\mathbf{P}^{\odot}\right)$ with respect to each upstream SU is regulated by power controller by using (4) as shown in Fig. 3. The rate demand depends on instant data rate $r_{i}^{\odot}(t)$ regulated by rate controller, which is nonlinear function of instant transmit power vector $\mathbf{P}^{\odot}$ according to (37) and (38).

\section{Simulation results}

\subsection{Simulation settings}

The simulation scenario is shown in Fig. 5, which consists of one bottleneck SU and $N=6$ randomly distributed upstream SUs transmitting data packets with elastic traffic towards bottleneck SU within a range of $100 \mathrm{~m} \times 100 \mathrm{~m}$. The scenario is easily extendable to a general case which involves much 


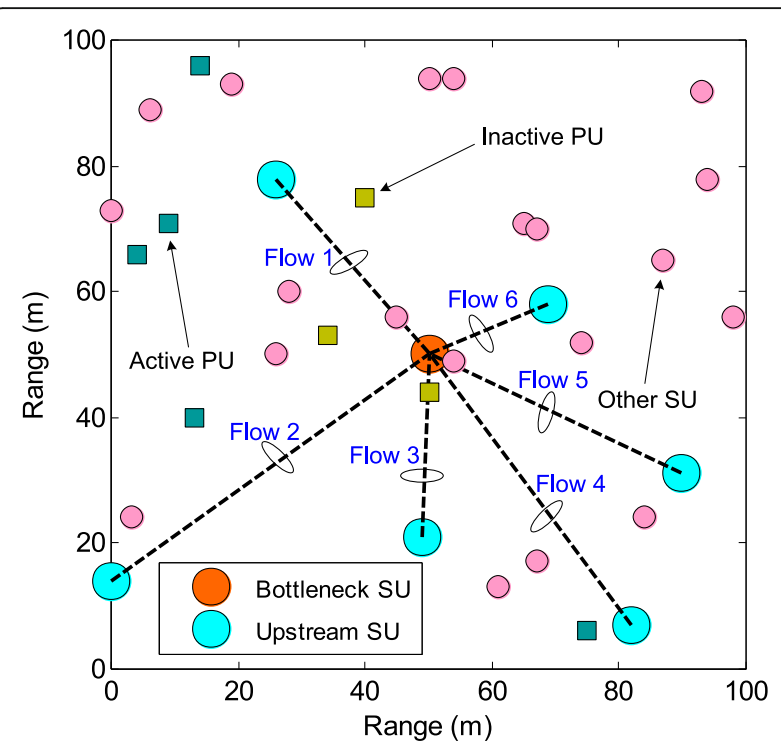

Fig. 5 Simulation scenario: $N=6$ upstream SUs transmitting data packets with elastic traffic towards bottleneck SU within a range of $100 \mathrm{~m} \times 100 \mathrm{~m}$. The dotted lines correspond to the successful transmission links from upstream SUs to bottleneck SU

more randomly distributed upstream SUs. Our simulations pay more attention to evaluate the effect of the cross-layer optimization framework for congestion and power control on both optimal data rate and optimal transmit power of six different per-link flows. Different from the channel with carrier frequency of $890.4 \mathrm{MHz}$ used by SUs, we assume that active PUs in simulation scenario occupy other uplink channels from set $\mathscr{H}$.This can make possible the successful transmissions of data packets from upstream SUs to bottleneck SU. The probability $\delta_{i}$ of uplink channels used by each $\mathrm{SU}$ is assumed to be 0.65 . The channel gain of link $l_{(i, b)}$ is defined with large-scale slow-fading model, given by $G_{(i, b)}=$ $100 g_{0}\left\|\vartheta_{i}(t)-\vartheta_{b}(t)\right\|^{-4}[34]$, where the reference channel gain $g_{0}$ is set to $9.7 \times 10^{-4}$ [44]. We adopt a processing gain factor $X=-1.5 / \log _{2}(5 \mathrm{BER})$ where the target bit error rate is $\mathrm{BER}=10^{-3}$ for multiple quadrature amplitude modulation with a symbol period of $\mathrm{T}_{s}=52.5 \mu \mathrm{s}$. The thermal noise power at bottleneck SU and interference caused by PBS are assumed to be $n_{0}=-50 \mathrm{dBm}$ and $I_{P}=10 \mathrm{dBm}$, respectively. In addition, the receiving reference power at bottleneck $\mathrm{SU}$ is chosen as $p_{(i, b)}^{r e f}=-37 \mathrm{dBm}$ for each upstream SU. With regard to the elastic traffic modeled by Poisson process [32], the flow arrival intensity is set to a normalized value $\lambda_{i}=125 \mathrm{bps}$ for each upstream $\mathrm{SU}$, and the mean of flow size is given as $\mathbb{E}\left[\lambda_{i}\right]=2 \mathrm{Mbits}$. Under our differential game models $\Gamma_{P L P C}$ and $\Gamma_{H H C C}$, we choose the pricing factor $\omega=22$, the penalty factor $\gamma=0.7$, two constant numbers $G_{2}=5.5$ and $G_{3}=360$.

Due to the lack of empirical data about available contact or all encounters between a pair of SUs, to evaluate the uncertainty of contact $\mathcal{C}^{(i, b)}$ along link $l_{(i, b)}$, we assume that the minimum and maximum values of encounter duration for all encounters within contact $\mathcal{C}^{(i, b)}$ is offered in Fig. 6. Note that Flow $i$ in Fig. 6 corresponds to contact $\mathcal{C}^{(i, b)}$ along link $l_{(i, b)}$, for $i=1,2, \cdots, 6$. We set the number of subintervals in Algorithm 1 to $M=8$ for all upstream SUs in that the number of encounters $K$ seems to be a lower value because of the short time interval in the simulations. In fact, game time or time interval is just set to $[0,5] \mathrm{s}$ in the following simulations. Under this setting, the probability distribution $\gamma^{(i, b)}$ of contact $\mathcal{C}^{(i, b)}$ derived from Algorithm 1 is assumed to comply with the contact distribution as provided by Fig. 7 .

The proposed OTPU algorithm for the PLPC problem is compared with the existing classical distributed constrained power control (DCPC) algorithm in [45]. The DCPC algorithm is a SINRconstrained power control algorithm which distributively and iteratively searches for transmit power updated from the $\varsigma$ th iteration to the $(\varsigma+1)$ th iteration. Let $\operatorname{SINR}_{i}^{\text {tar }}$ denote the target SINR for upstream SU $v_{i}$ to maintain a certain QoS requirement. In the simulations, the target SINR can be set as $\operatorname{SINR}_{i}^{\text {tar }}=8 d B$. Therefore, the iterative function of transmit power update in the DCPC algorithm with number of iteration $\varsigma=0,1,2, \cdots$ is specifically given as [45]:

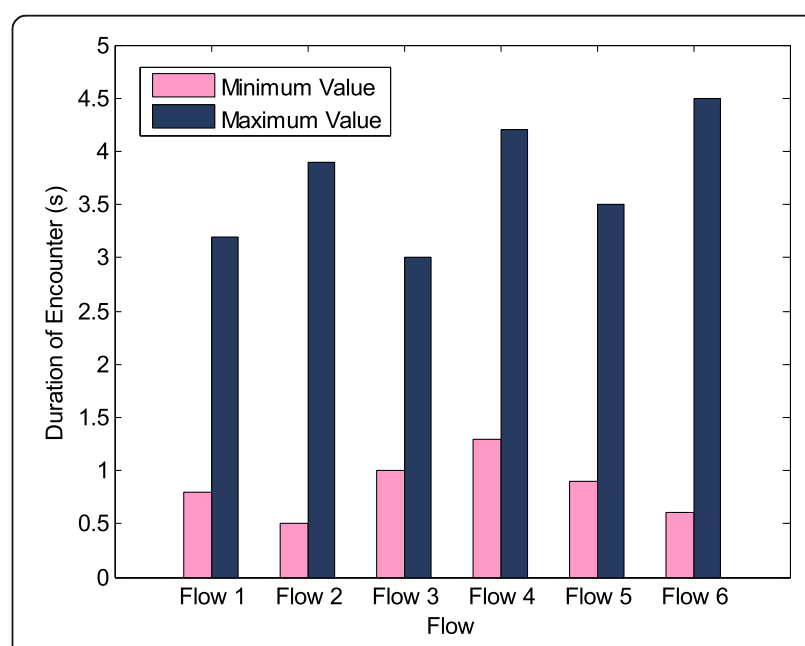

Fig. 6 Comparison between minimum value and maximum value of encounter duration among different per-link flows 

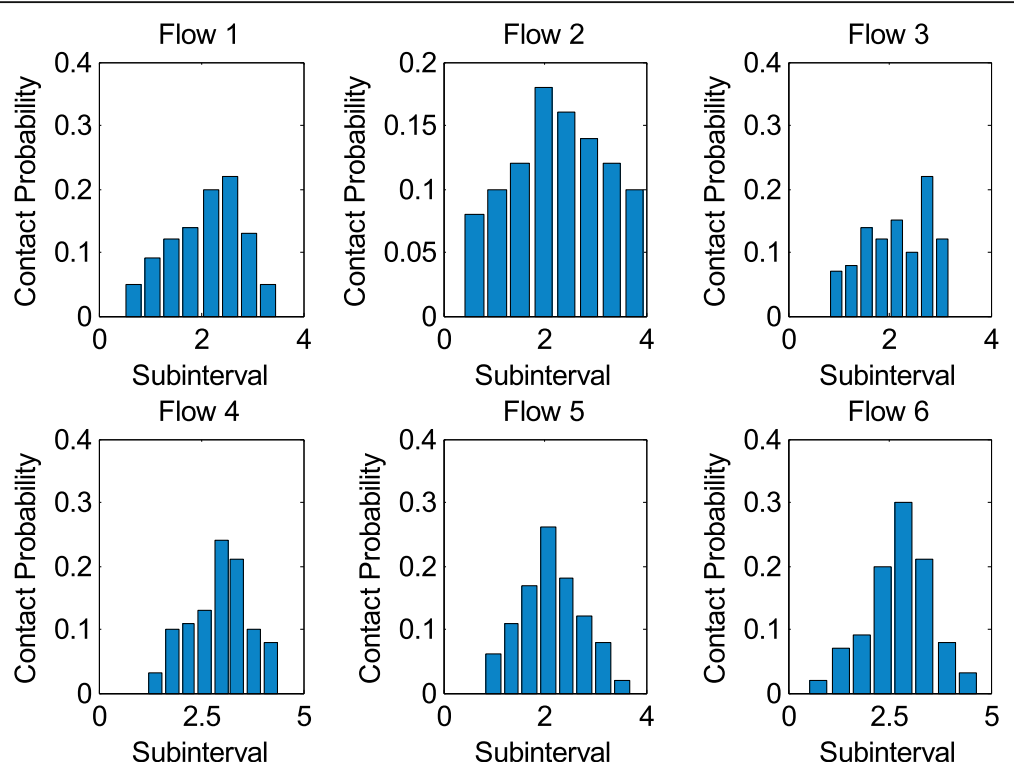

Fig. 7 Probability distribution of contact between upstream SU and bottleneck SU under different per-link flows when the number of subintervals $M=8$

$$
p_{i}^{(\varsigma+1)}=\min \left\{\bar{p}_{i}, \frac{\operatorname{SINR}_{i}^{\text {tar }}}{\operatorname{SINR}_{(i, b)}^{(\varsigma)}(\mathbf{P})} \cdot p_{i}^{(\varsigma)}\right\} .
$$

\section{Results}

\subsection{Performance of OTPU algorithm}

Figure 8 shows the optimal transmit power comparison for six per-link flows between the OTPU algorithm with the evolution of discount factor $a$ and the DCPC algorithm with $\varsigma=300$ iterations. This figure clearly depicts that an increased discount factor from 0.1 to 0.9 will increase the optimal transmit power of each flow under the OTPU algorithm. Apparently, this is a direct consequence of discount factor $a$ on the optimal transmit power according to (25). However, it is observed that the optimal transmit power of each flow via the DCPC algorithm presents a fixed constant value. This is due to the fact that the optimal transmit power of each flow via the DCPC algorithm converges to an expected equilibrium point after 300 iterations. It is worth mentioning that Theorem 1 mathematically makes the optimal transmit power of each upstream SU converge to a Nash equilibrium point distributively. From the results, we can also see that the optimal transmit power of each flow by the OTPU algorithm is obviously lower than that of the DCPC algorithm. This can be explained by the fact that DCPC algorithm gives rise to more power consumption for maintaining a certain SINR for each upstream SU. However, the optimal transmit power of each flow based on the OTPU algorithm mainly depends upon the maximum transmit power threshold of upstream SU. On the other hand, the instant power level can be further reduced via the change of discount factor $a$.

\subsection{The impact of discount factor on optimal transmit power}

Figure 9 illustrates the optimal transmit power comparison for six per-link flows via the OTPU algorithm under different discount factors. It is noted that the total interference caused by six upstream SUs satisfies the interference temperature limit $\varpi_{\mathrm{PBS}}=-10 \mathrm{dBm}$ according to the constraint of (6). As the discount factor increases, the optimal transmit power of six flows obtained by the OTPU algorithm will raise as well. As expected, the optimal transmit power of flow 6 can achieve the minimum transmit power level with approximately $50 \mathrm{~mW}$, and the optimal transmit power of flow 2 can obtain higher transmit power level with the maximum value nearly $570 \mathrm{~mW}$. However, the increasing rate of the optimal transmit power in regard to flows 6 , 3 , and 1 flattens out after discount factor $a=0.6$. The reason is as follows. Firstly, based on (25), the discount factor effect is in direct proportion to the optimal transmit power. One the other hand, with even higher Euclidean distance between upstream 


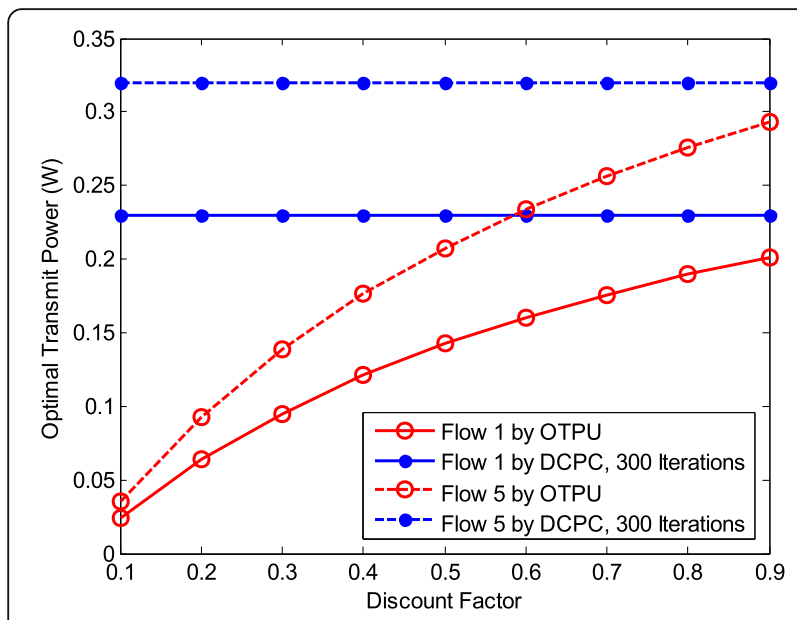

a

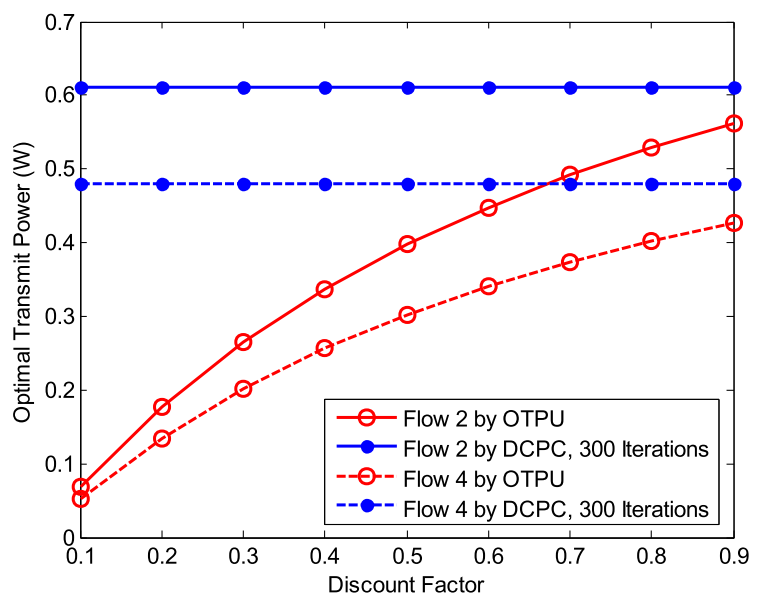

b

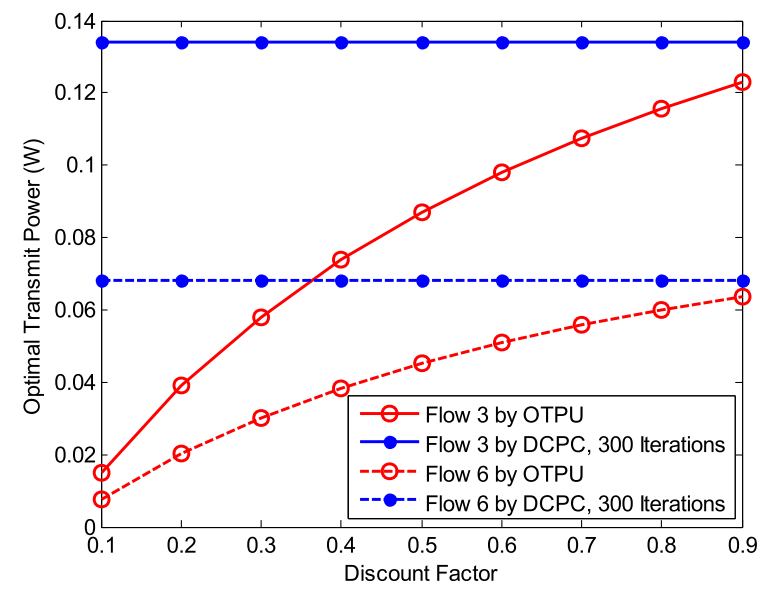

C

Fig. 8 Optimal transmit power comparison between our proposed OTPU algorithm and DCPC algorithm with 300 iterations under different per-link flows: a Flows 1 and 5, b flows 2 and 4, and c flows 3 and 6

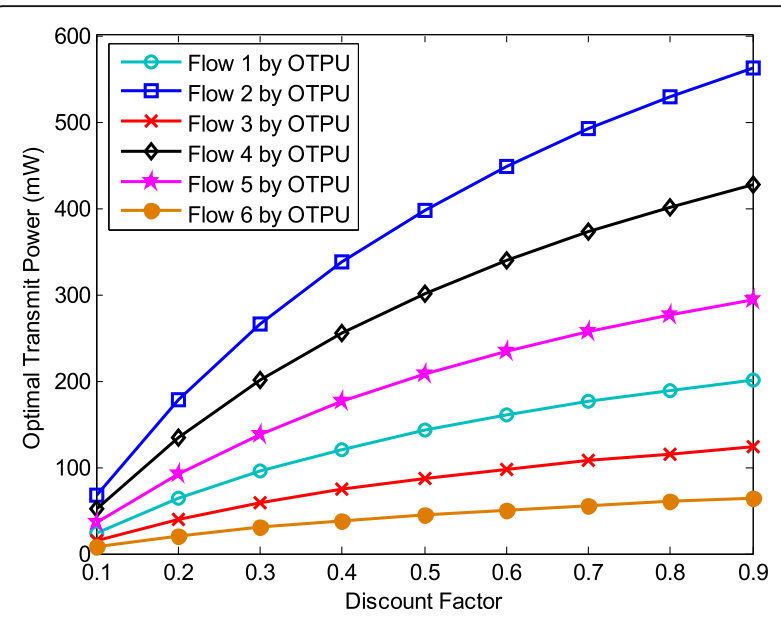

Fig. 9 Optimal transmit power comparison among six per-link flows through our proposed OTPU algorithm under interference temperature limit $\omega_{\mathrm{PBS}}=-10 \mathrm{dbm}$

SU and bottleneck SU, the maximum transmit power threshold will increase as well according to (11). Under the simulation scenario, the higher Euclidean distance of the successful transmission link of flow 2 leads to a higher transmit power level accordingly.

\subsection{Optimal data rate performance of ODRU algorithm}

Figure 10 exhibits the evolution of the optimal data rate for six per-link flows obtained by the ODRU algorithm versus game time $t \in[0,5] \mathrm{s}$ under the condition of discount factor $\tau=0.2$ and saturation value $\hat{L}_{b}=1 \mathrm{Mbps}$. From the results, we can see the optimal data rate for six flows gradually increase with the growth of game time $t$. Meanwhile, the optimal data rate levels of six flows are very close from 0 to $4 \mathrm{~s}$. When game time $t$ is more than $4 \mathrm{~s}$, the gaps among the optimal data rate levels will be enlarged. This demonstrates that the optimal data rate has large values during the game time of the end interval of the game. Under discount factor $\tau=0.2$, the optimal data rate value of flow 2 is much larger than those of other flows with maximum value of $300 \mathrm{kbps}$, and the optimal rate of flow 6 has the lowest level within $50 \mathrm{kbps}$. It can also be observed that the optimal data rate of flow 2 yields significant performance gains than other flows under the condition of the fixed discount factor. According to saturation value $\hat{L}_{b}=1 \mathrm{Mbps}$, we can observe that the total data rate generated by six upstream SUs is subject to the buffer constraint $\phi_{b}(t) \leq \hat{L}_{b}$ such that the instant data rate levels should not be adjusted through the ODRU algorithm. 


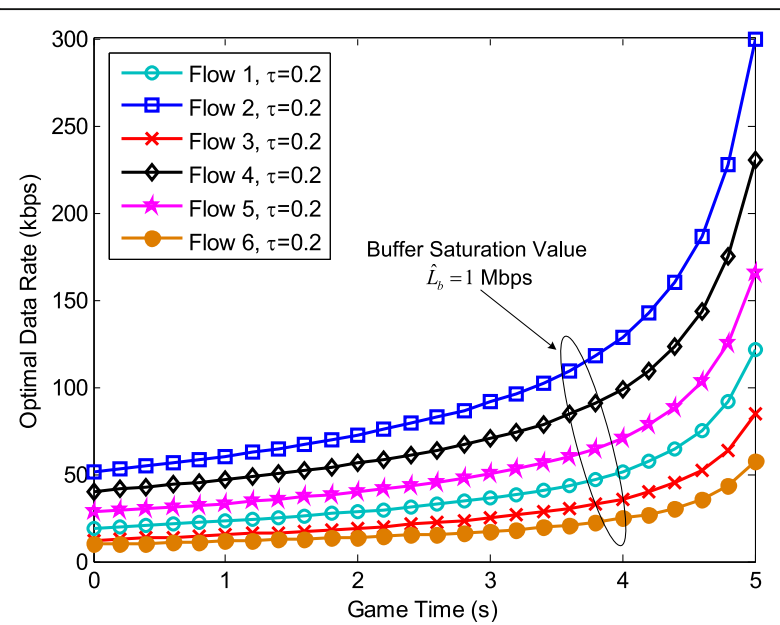

Fig. 10 Optimal data rate comparison among six per-link flows through our proposed ODRU algorithm under saturation value $\hat{L}_{b}=1$ Mbps

Figure 11 depicts the optimal data rate update comparison for six per-link flows with the aid of the ODRU algorithm on the condition of discount factor $\tau=0.2$ and saturation value $\hat{L}_{b}=490 \mathrm{kbps}$. It is implicitly revealed that the total data rate caused by six upstream SUs fail to guarantee the buffer constraint $\phi_{b}(t) \leq \hat{L}_{b}$ such that the instant data rate levels must be updated according to the ODRU algorithm. Hence, the evolution of the optimal data rate levels of six upstream SUs will enter the rate update zone (i.e., shadow area in Fig. 11) when the constraint $\phi_{b}(t)>\hat{L}_{b}$. From the results, we can see that the large values of the optimal data rate have been considerably dwindled according to the distributed rate update function in (41) when instant game time $t=4.3 \mathrm{~s}$ in order to meet the buffer constraint of bottleneck SU.

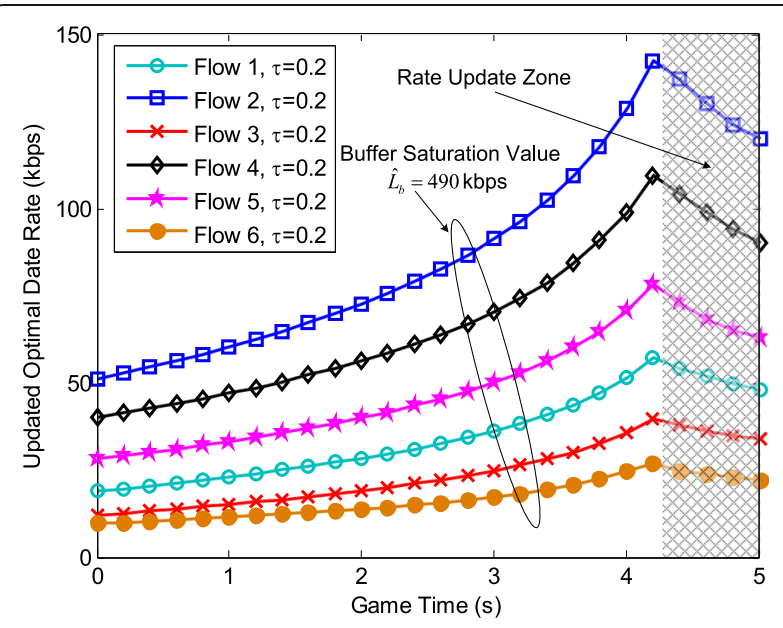

Fig. 11 Optimal data rate comparison among six per-link flows through our proposed ODRU algorithm under saturation value $\hat{L}_{b}=490 \mathrm{kbps}$
6.4 The impact of discount factor on optimal data rate Figure 12 displays the evolution of the optimal data rate for six per-link flows via the ODRU algorithm versus discount factor $\tau$ under the condition of two fixed instant game time point $t$ (i.e., $t=3$ and $t=4$ ) and saturation value $\hat{L}_{b}=950 \mathrm{kbps}$. From the results, we can see the total data rate generated by six upstream SUs accommodates for the buffer constraint $\phi_{b}(t) \leq \hat{L}_{b}$. It can be also observed that as the discount factor increases from 0.1 to 0.9 , the optimal data rate of six flows obtained by the ODRU algorithm will decrease accordingly. The reason for this is that the utility function of each upstream SU must be discounted by the factor $e^{-\tau t}$ at time $t$ under the differential game structure $\Gamma_{H H C C}$. As we expected, the optimal data rate of flow 6 can obtain the minimum rate level within approximately interval $[6,26] \mathrm{kbps}$, and the optimal data rate of flow 2 can gain the maximum value of data rate with nearly interval [30, 140] kbps. This can be explained by the fact that the higher Euclidean distance of the successful transmission link of flow 2 will result in a higher transmit power level accordingly. This result of higher transmit power level of flow 2 will lead to the more link capacity supply. It implies that the upstream SU has the enough link capacity supply to achieve higher data rate in the proposed cross-layer optimization framework. Essentially, this signifies the importance of cross-layer coordination mechanism on the coupling between rate demand regulated by rate controller and capacity supply regulated by power controller.

\section{Conclusions}

In this paper, a distributed cross-layer optimization framework for congestion and power control for CRANETs under predictable contact has been proposed. Particularly, we introduced a predictable contact model by achieving the probability distribution of contact between any pair of SUs, aiming to measure the uncertainty of contact. Also, an entropy paradigm was presented to characterize quantitatively the probability distribution of contact. We employed a differential game theoretic approach to formulate the PLPC problem and the HHCC problem, and obtained the optimal transmit power and the optimal data rate of upstream SUs via dynamic programming and maximum principle. To guarantee the interference power constraint for active PUs and the buffer constraint of bottleneck SU, we developed two distributed update algorithms to locally adjust optimal transmit power and optimal data rate of upstream SUs. Finally, we presented a distributed implementation strategy to construct the cross-layer optimization framework for 

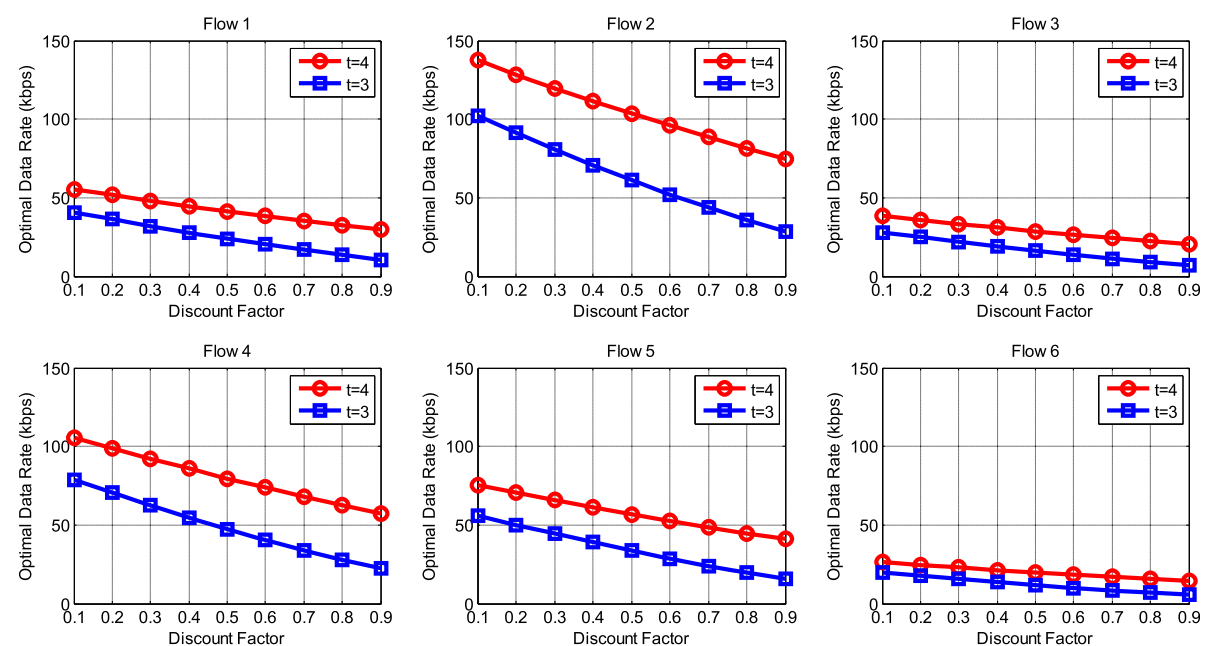

Fig. 12 Optimal data rate comparison among six per-link flows through our proposed ODRU algorithm for different discount factors under saturation value $\hat{L}_{b}=950 \mathrm{kbps}$

congestion and power control by jointly optimizing PLPC-HHCC simultaneously and validated its performance with simulations. What we have discussed in this paper is the portion of foundation for the cross-layer optimization framework in CRANETs. In the future work, a joint objective function to achieve congestion and power control will be considered. Moreover, it will be interesting and important to investigate a trade-off parameter as a whole to reflect the benefits of the proposed framework.

\section{Appendix 1}

\subsection{Proof of Theorem 1}

According to the dynamic optimization problem $\mathbb{P} 1$, performing the maximization operation of the right hand side of (24) with respect to $p_{i}(t)$ yields the following optimal solution:

$$
p_{i}^{\#}(t)=\bar{p}_{i}+\frac{C_{(i, b)}^{*}(\overline{\mathbf{P}})}{2} \frac{\partial V^{i}\left(p_{i}, I\right)}{\partial I} .
$$

Substituting $p_{i}^{\#}(t)$ in (45) into (24), we obtain:

$$
\begin{aligned}
a V^{i}\left(p_{i}^{\#}, I\right)= & \frac{C_{(i, b)}^{*}(\overline{\mathbf{P}})}{4}\left(\frac{\partial V^{i}\left(p_{i}^{\#}, I\right)}{\partial I}\right)^{2}-\omega I(t) \\
& +\frac{\partial V^{i}\left(p_{i}^{\#}, I\right)}{\partial I}\left(\sum_{i \in \mathcal{N}}\left(\bar{p}_{i}+\frac{C_{(i, b)}^{*}(\overline{\mathbf{P}})}{2} \frac{\partial V^{i}\left(p_{i}^{\#}, I\right)}{\partial I}\right)-\gamma \cdot I(t)\right) .
\end{aligned}
$$

Upon solving the differential equation in (46), $V^{i}\left(p_{i}^{\#}, I\right)$ can be easily shown to be equivalent to the following equation:

$$
V^{i}\left(p_{i}^{\#}, I\right)=\frac{\omega}{a(a+\gamma)}\left(\frac{\omega C_{(i, b)}^{*}(\overline{\mathbf{P}})(1+2 N)}{4(a+\gamma)}-a I-\sum_{i \in \mathcal{N}} \bar{p}_{i}\right) .
$$

Thus, an optimal transmit power $p_{i}^{\#}(t)$ which constitutes a Nash equilibrium solution to $\mathbb{P} 1$ is given by:

$$
p_{i}^{\#}(t)=\bar{p}_{i}-\frac{\omega C_{(i, b)}^{*}(\overline{\mathbf{P}})}{2(a+\gamma)} .
$$

Then, we can obtain the expression of $p_{i}^{\#}(t)$ and $V^{i}\left(p_{i}\right.$, I) as given by Theorem 1 .

\section{Appendix 2}

\subsection{Proof of Proposition 1}

By substituting the vector of optimal transmit power $\mathbf{P}^{\#}$ $=\left\{p_{1}^{\#}(t), p_{2}^{\#}(t), \cdots, p_{N}^{\#}(t)\right\}$ into the interference power constraint inequality in (6), we can obtain:

$$
\sum_{i \in \mathcal{N}} p_{i}^{\#}(t) G_{(i, b)} \leq \varpi_{\mathrm{PBS}} .
$$

After taking the logarithm of both sides of (49), we have:

$$
\sum_{i \in \mathcal{N}} \log _{2}\left(p_{i}^{\#}(t) G_{(i, b)}\right) \leq \log _{2} \varpi_{\mathrm{PBS}} .
$$

Through rearranging terms, we have:

$$
\log _{2} \prod_{i \in \mathcal{N}} p_{i}^{\#}(t)+\log _{2} \prod_{i \in \mathcal{N}} G_{(i, b)} \leq \log _{2} \varpi_{\mathrm{PBS}} .
$$

By taking into account the large-scale slow-fading channel model to describe the wireless transmission 
environment, the channel gain of link from upstream SU $v_{i}$ to bottleneck SU $v_{b}$ can be formulated as [30]:

$$
G_{(i, b)}=g_{0}\left(\frac{\left\|\vartheta_{i}(t)-\vartheta_{b}(t)\right\|}{100}\right)^{-4}
$$

where $g_{0}$ is a reference channel gain at a distance of $100 \mathrm{~m}$ [34]. We substitute $G_{(i, b)}$ in (52) into (51) and then we have:

$$
\log _{2} \prod_{i \in \mathcal{N}} p_{i}^{\#}(t) \leq \log _{2} \frac{\varpi_{\mathrm{PBS}}}{\prod_{i \in \mathcal{N}} g_{0}\left(\frac{\left\|\vartheta_{i}(t)-\vartheta_{b}(t)\right\|}{100}\right)^{-4}} .
$$

Thus, (53) can be rewritten as follows:

$$
\prod_{i \in \mathcal{N}} p_{i}^{\#}(t) \leq \frac{\varpi_{\mathrm{PBS}}}{\left(10^{6} g_{0}\right)^{N}} \prod_{i \in \mathcal{N}}\left\|\vartheta_{i}(t)-\vartheta_{b}(t)\right\|^{4}
$$

Through defining $G_{1}=\varpi_{\mathrm{PBS}} /\left(10^{6} g_{0}\right)^{N}$, we can easily have the solution of (27).

\section{Appendix 3}

\subsection{Proof of Theorem 2}

According to the dynamic optimization problem $\mathbb{P} 2$, by performing the maximization operation of the right hand side of (30) with respect to $r_{i}(t)$, we can obtain:

$$
r_{i}^{\#}(t)=\frac{e^{\tau t}}{2 B_{(i, b)}} \frac{\partial W^{i}\left(r_{i}, x\right)}{\partial x}
$$

Substituting $W^{i}\left(r_{i}, x\right) \triangleq\left(Y_{i}(t) x(t)+J_{i}(t)\right) e^{-\tau t}$ and $r_{i}^{\#}(t)$ in (55) into (30), we have:

$$
\begin{aligned}
r_{i}^{\#}(t)= & \frac{Y_{i}(t) x(t)}{2 B_{(i, b)}}, \\
\frac{\mathrm{d} Y_{i}(t)}{\mathrm{d} t}= & \frac{1}{4 B_{(i, b)}} Y_{i}^{2}(t)+(\tau-1) Y_{i}(t)-A_{(i, b)} \\
& +\frac{Y_{i}(t)}{2} \sum_{j \in \mathcal{N} \backslash i} \frac{Y_{j}(t)}{B_{(j, b)}}, \\
\frac{\mathrm{d} J_{i}(t)}{\mathrm{d} t}= & \tau \cdot J_{i}(t) .
\end{aligned}
$$

Hence, this completes the proof.

\section{Appendix 4}

\subsection{Proof of Proposition 2}

Owing to the symmetric form of $Y_{i}(t)$ and $Y_{j}(t)$ in (57), we can immediately denote (57) by Riccati equation:

$$
\begin{aligned}
\frac{\mathrm{d} Y_{i}(t)}{\mathrm{d} t}= & \left(\frac{1}{4 B_{(i, b)}}+\frac{1}{2} \sum_{j \in \mathcal{N} \backslash i} \frac{1}{B_{(j, b)}}\right) Y_{i}^{2}(t) \\
& +(\tau-1) Y_{i}(t)-A_{(i, b)} .
\end{aligned}
$$

As such, the form of (59) can be rearranged by differential equation as:

$$
\begin{aligned}
\wp_{1}\left(Y_{i}(t), t\right) \mathrm{d} t+ & \wp_{2}\left(Y_{i}(t), t\right) \mathrm{d} Y_{i}(t)=0, \\
\wp_{1}\left(Y_{i}(t), t\right)= & \left(\frac{1}{4 B_{(i, b)}}+\frac{1}{2} \sum_{j \in \mathcal{N} \backslash i} \frac{1}{B_{(j, b)}}\right) Y_{i}^{2}(t) \\
& +(\tau-1) Y_{i}(t)-A_{(i, b)},
\end{aligned}
$$

$$
\wp_{2}\left(Y_{i}(t), t\right)=-1 .
$$

Recall that we define $\Omega_{(i, b)}=1 /\left(4 B_{(i, b)}\right)+0.5 \sum_{j \in \mathcal{N} \backslash i} 1$ $/ B_{(j, b)}$, for $4 \Omega_{(i, b)} A_{(i, b)}+(\tau-1)^{2}>0$. Next, we turn to present a non-zero integrating factor $\mathfrak{S}\left(Y_{i}(t), t\right)$ that can make equation in (61) an exact form by multiplying it on both sides of (61). Here, we can easily obtain:

$$
\mathfrak{I}\left(Y_{i}(t), t\right)=\frac{1}{\left|\Omega_{(i, b)} Y_{i}^{2}(t)+(\tau-1) Y_{i}(t)-A_{(i, b)}\right|} .
$$

So, (63) multiplied by $\mathfrak{S}\left(Y_{i}(t), t\right)$ is exact, and then we obtain:

$$
\frac{\partial \mathfrak{I}\left(Y_{i}(t), t\right)}{\partial Y_{i}(t)}=-\frac{1}{\left|\Omega_{(i, b)} Y_{i}^{2}(t)+(\tau-1) Y_{i}(t)-A_{(i, b)}\right|},
$$

$$
\frac{\partial \mathfrak{I}\left(Y_{i}(t), t\right)}{\partial t}=1 .
$$

When $\Omega_{(i, b)} Y_{i}^{2}(t)+(\tau-1) Y_{i}(t)>A_{(i, b)}$, we can easily define $\varepsilon=\sqrt{(\tau-1)^{2}+4 \Omega_{(i, b)} A_{(i, b)}}$. By integrating (64) and (65) with respect to $Y_{i}(t)$, we have:

$$
\begin{aligned}
\mathfrak{J}\left(Y_{i}(t), t\right)= & -\frac{1}{\varepsilon} \ln \left|\frac{2 \Omega_{(i, b)} Y_{i}(t)+(\tau-1)-\varepsilon}{2 \Omega_{(i, b)} Y_{i}(t)+(\tau-1)+\varepsilon}\right| \\
& +h(t), \\
\frac{\partial \mathfrak{J}\left(Y_{i}(t), t\right)}{\partial t}= & \frac{\mathrm{d} h(t)}{\mathrm{d} t} .
\end{aligned}
$$

We can easily obtain $h(t)=t$. Let $\mathfrak{S}\left(Y_{i}(t), t\right)=G_{2}$, where $G_{2}$ is a constant number. Upon solving (66) as follows:

$$
t-\frac{1}{\varepsilon} \ln \left|\frac{2 \Omega_{(i, b)} Y_{i}(t)+(\tau-1)-\varepsilon}{2 \Omega_{(i, b)} Y_{i}(t)+(\tau-1)+\varepsilon}\right|=G_{2} .
$$

Solving the above equation in (68) with respect to $Y_{i}(t)$, yields the desired result in (35). 


\section{Appendix 5}

\subsection{Proof of Proposition 3}

For vector $\mathbf{P}^{\#}$, it is clear that the received SINR of link $l_{(i, b)}$ at bottleneck SU $v_{b}$ satisfies the following inequality when all the upstream SUs with the equal Euclidean distance to bottleneck SU $v_{b}$ :

$$
\begin{aligned}
\operatorname{SINR}_{(i, b)}\left(\mathbf{P}^{\#}\right) & \leq \frac{p_{i}^{\#}(t) G_{(i, b)}}{\sum_{j \in \mathcal{N} \backslash i} p_{j}^{\#}(t) G_{(j, b)}} \\
& =\frac{p_{i}^{\#}(t)}{\sum_{j \in \mathcal{N} \backslash i} p_{j}^{\#}(t)} .
\end{aligned}
$$

We can also approximate logarithmic function $\log _{2}(\cdot)$ by:

$$
\log _{2}\left(1+x \cdot \operatorname{SINR}_{(i, b)}\left(\mathbf{P}^{\#}\right)\right) \approx \log _{2}\left(x \cdot \operatorname{SINR}_{(i, b)}\left(\mathbf{P}^{\#}\right)\right) .
$$

Thus, shadow price $\boldsymbol{N}_{i}$ should be subject to a strict lower bound:

$$
\begin{aligned}
\boldsymbol{\aleph}_{i} \approx & \left(\frac{1}{\log _{2}\left(X \cdot \operatorname{SINR}_{(i, b)}\left(\mathbf{P}^{\#}\right)\right)}\right) \frac{r_{i}^{\#}(t)}{x^{\#}(t)} \\
& \geq\left(\log _{2}\left(X \cdot \frac{p_{i}^{\#}(t)}{\sum_{j \in \mathcal{N} \backslash i} p_{j}^{\#}(t)}\right)\right)^{-1} \frac{r_{i}^{\#}(t)}{x^{\#}(t)} .
\end{aligned}
$$

which coincides with (41).

\begin{abstract}
Abbreviations
BER: Bit error rate; CR: Cognitive radio; CRANET: Cognitive radio ad hoc network; CRN: CR network; DCPC: Distributed constrained power control; HHCC: Hop-by-hop congestion control; ODRU: Optimal data rate update; OTPU: Optimal transmit power update; PBS: Primary base station; PLPC: Perlink power control; PU: Primary user; QoS: Quality of service; RTT: Round trip time; SINR: Signal-to-interference-plus-noise ratio; SU: Secondary user; TCP: Transmission control protocol; UDP: User datagram protocol
\end{abstract}

\section{Acknowledgements}

We are grateful to the anonymous reviewers for their valuable comments and suggestions that have improved the paper.

\section{Funding}

This work was supported in part by the National Natural Science Foundation of China under Grants 61402147, 61402001, and 61501406; the Research Program for Top-notch Young Talents in Higher Education Institutions of Hebei Province, China, under Grant BJ2017037; the Research and Development Program for Science and Technology of Handan, China, under Grant 1621203037; and the Natural Science Foundation of Hebei Province of China under Grant F2017402068.

\section{Availability of data and materials}

Not applicable.

\section{Authors' contributions}

LZ conceived the idea of this work and wrote the paper. HX provided valuable insights for the scheme and reviewed the manuscript. LZ and FZ performed the simulations and revised the paper. All authors read and approved the final manuscript.

\section{Competing interests}

The authors declare that they have no competing interests.

\section{Publisher's Note}

Springer Nature remains neutral with regard to jurisdictional claims in published maps and institutional affiliations.

\section{Author details}

${ }^{1}$ School of Information and Electrical Engineering, Hebei University of Engineering, Handan 056038, China. ${ }^{2}$ School of Computer and Communication Engineering, University of Science and Technology Beijing, Beijing 100083, China.

Received: 22 September 2017 Accepted: 22 February 2018 Published online: 13 March 2018

\section{References}

1. S Haykin, Cognitive radio: brain-empowered wireless communications. IEEE J. Sel. Areas Commun. 23(2), 201-220 (2005)

2. Z Z Ding, Y Liu, J Choi, Q Sun, M Elkashlan, I Chih-Lin, HV Poor, Application of non-orthogonal multiple access in LTE and $5 \mathrm{G}$ networks. IEEE Commun. Mag. 55(2), 185-191 (2017)

3. V Rakovic, D Denkovski, V Atanasovski, P Mahonen, L Gavrilovska, Capacityaware cooperative spectrum sensing based on noise power estimation. IEEE Trans. Commun. 63(7), 2428-2441 (2015)

4. N Zhang, $\mathrm{H}$ Liang, N Cheng, Dynamic spectrum access in multi-channel cognitive radio networks. IEEE J. Sel. Areas Commun. 32(11), 2053-2064 (2014)

5. PK Sharma, PK Upadhyay, Performance analysis of cooperative spectrum sharing with multiuser two-way relaying over fading channels. IEEE Trans. Veh. Technol. 66(2), 1324-1333 (2017)

6. IF Akyildiz, W-Y Lee, KR Chowdhury, CRAHNs: cognitive radio ad hoc networks. Ad Hoc Netw. 7(5), 810-836 (2009)

7. A Goldsmith, SA Jafar, I Maric, S Srinivasa, Breaking spectrum gridlock with cognitive radios: an information theoretic perspective. Proc. IEEE 97(5), 894-914 (2009)

8. S Gong, P Wang, Y Liu, W Zhuang, Robust power control with distribution uncertainty in cognitive radio networks. IEEE J. Sel. Areas Commun. 31(11), 2397-2408 (2013)

9. G Ozcan, MC Gursoy, Optimal power control for underlay cognitive radio systems with arbitrary input distributions. IEEE Trans. Wirel. Commun. 14(8), 4219-4233 (2015)

10. S Parsaeefard, AR Sharafat, Robust distributed power control in cognitive radio networks. IEEE Trans. Mobile Comput. 12(4), 609-620 (2013)

11. S-C Lin, K-C Chen, Spectrum-map-empowered opportunistic routing for cognitive radio ad hoc networks. IEEE Trans. Veh. Technol. 63(6), 2848-2861 (2014)

12. J Wang, H Yue, L Hai, Y Fang, Spectrum-aware anypath routing in multi-hop cognitive radio networks. IEEE Trans. Mobile Comput. 16(4), 1176-1187 (2017)

13. J Zhao, G Cao, Spectrum-aware data replication in intermittently connected cognitive radio networks (Proc. IEEE INFOCOM, Toronto, 2014), pp. 2238-2246

14. V Cerf, S Burleigh, L Torgerson, R Durst, K Scott, K Fall, H Weiss, Delaytolerant network architecture: The evolving Interplanetary Internet (InternetDraft, IPN Research Group, 2002). https://tools.ietf.org/html/rfc4838

15. KR Chowdhury, M Di Felice, IF Akyildiz, TCP CRAHN: a transport control protocol for cognitive radio ad hoc networks. IEEE Trans. Mobile Comput. 12(4), 790-803 (2013)

16. D Sarkar, H Narayan, Transport layer protocols for cognitive networks (Proc. IEEE INFOCOM, San Diego, 2010), pp. 1-6

17. B Soelistijanto, MP Howarth, Transfer reliability and congestion control strategies in opportunistic networks: a survey. IEEE Commun. Surveys \& Tutorials 16(1), 538-555, First Quarter (2014)

18. AK Al-Ali, K Chowdhury, TFRC-CR: an equation-based transport protocol for cognitive radio networks. Ad Hoc Netw. 11(6), 1836-1847 (2013)

19. K Tsukamoto, S Koba, M Tsuru, Y Oie, Cognitive radio-aware transport protocol for mobile ad hoc networks. IEEE Trans. Mobile Comput. 14(2), 288-301 (2015)

20. M Chiang, Balancing transport and physical layers in wireless multihop networks: jointly optimal congestion control and power control. IEEE J. Sel. Areas Commun. 23(1), 104-116 (2005)

21. HJ Lee, JT Lim, Cross-layer congestion control for power efficiency over wireless multihop networks. IEEE Trans. Veh. Technol. 58(9), 5274-5278 (2009)

22. Q-V Pham, W-J Hwang, Network utility maximization-based congestion control over wireless networks: a survey and potential directives. IEEE Commun. Surveys \& Tutorials 19(2), 1173-1200, Second Quarter (2017) 
23. Q Gao, J Zhang, SV Hanly, Cross-layer rate control in wireless networks with lossy links: leaky-pipe flow, effective network utility maximization and hop-by-hop algorithms. IEEE Trans. Wirel. Commun. 8(6), 3068-3076 (2009)

24. Q-V Pham, W-J Hwang, Network utility maximization in multipath lossy wireless networks. International J. of Commun. Syst. 30(5), e3094 (2017)

25. S Guo, C Dang, Y Yang, Joint optimal data rate and power allocation in lossy mobile ad hoc networks with delay-constrained traffics. IEEE Trans. Comput. 64(3), 747-762 (2015)

26. KXiao, S Mao, JK Tugnait, MAQ: a multiple model predictive congestion control scheme for cognitive radio networks. IEEE Trans. Wirel. Commun. 16(4), 2614-2626 (2017)

27. Y Song, J Xie, End-to-end congestion control in multi-hop cognitive radio ad hoc networks: to timeout or not to timeout? (Proc. IEEE Globecom Workshops, Atlanta, 2013), pp. 1-6

28. X Zhong, Y Qin, L Li, TCPNC-DGSA: efficient network coding scheme for TCP in multi-hop cognitive radio networks. Wireless Pers. Commun. 84(2), 1243-1263 (2015)

29. A Cammarano, FL Presti, G Maselli, L Pescosolido, C Petrioli, Throughputoptimal cross-layer design for cognitive radio ad hoc networks. IEEE Trans. Parallel Distrib. Syst. 26(9), 2599-2609 (2015)

30. MV Nguyen, CS Hong, S Lee, Cross-layer optimization for congestion and power control in OFDM-based multi-hop cognitive radio networks. IEEE Trans. Commun. 60(8), 2101-2112 (Aug. 2012)

31. MV Nguyen, S Lee, S You, CS Hong, LB Le, Cross-layer design for congestion, contention, and power control in CRAHNs under packet collision constraints. IEEE Trans. Wirel. Commun. 12(11), 5557-5571 (2013)

32. A Alshamrani, $X$ Shen, $L-L X i e$, QoS provisioning for heterogeneous services in cooperative cognitive radio networks. IEEE J. Sel. Areas Commun. 29(4), 819-830 (2011)

33. SB Fredj, T Bonald, A Proutiere, G Regnie, JW Roberts, Statistical bandwidth sharing: A study of congestion at flow level (Proc. ACM SIGCOMM, San Diego, 2001), pp. 111-122

34. NH Tran, CS Hong, S Lee, Cross-layer design of congestion control and power control in fast-fading wireless networks. IEEE Trans. Parallel Distrib. Syst. 24(2), 260-274 (2013)

35. H Zhang, S Huang, C Jiang, K Long, VCM Leung, HV Poor, Energy efficient user association and power allocation in millimeter-wave-based ultra dense networks with energy harvesting base stations. IEEE J. Sel. Areas Commun. 35(9), 1936-1947 (2017)

36. H Zhang, Y Nie, J Cheng, VCM Leung, A Nallanathan, Sensing time optimization and power control for energy efficient cognitive small cell with imperfect hybrid spectrum sensing. IEEE Trans. Wirel. Commun. 16(2), 730-743 (2017)

37. S Kandukuri, S Boyd, Optimal power control in interference-limited fading wireless channels with outage-probability specifications. IEEE Trans. Wirel. Commun. 1(1), 46-55 (2002)

38. A Khelil, PJ Marron, K Rothermel, Contact-based mobility metrics for delaytolerant ad hoc networking (Proc. IEEE MASCOTS, Atlanta, 2005), pp. 1-10

39. JC Principe, Information theoretic learning: Renyi's entropy and Kernel perspectives (Springer, New York, 2010)

40. F Heliot, MA Imran, R Tafazolli, On the energy efficiency-spectral efficiency trade-off over the MIMO Rayleigh fading channel. IEEE Trans. Commun. 60(5), 1345-1356 (2012)

41. H Zhang, C Jiang, NC Beaulieu, X Chu, X Wang, TQS Quek, Resource allocation for cognitive small cell networks: a cooperative bargaining game theoretic approach. IEEE Trans. Wirel. Commun. 14(6), 3481-3493 (2015)

42. DWK Yeung, LA Petrosyan, Cooperative stochastic differential games (Springer, New York, 2005)

43. H Zhang, H Liu, J Cheng, VCM Leung, Downlink energy efficiency of power allocation and wireless backhaul bandwidth allocation in heterogeneous small cell networks. IEEE Trans. Commun. PP(99), 1-1 (2017)

44. C-G Yang, J-D Li, Z Tian, Optimal power control for cognitive radio networks under coupled interference constraints: a cooperative game-theoretic perspective. IEEE Trans. Veh. Technol. 59(4), 1696-1706 (2010)

45. Y Xing, CN Mathur, MA Haleem, R Chandramouli, KP Subbalakshmi, Dynamic spectrum access with QoS and interference temperature constraints. IEEE Trans. Mobile Comput. 6(4), 423-433 (2007)

\section{Submit your manuscript to a SpringerOpen ${ }^{\circ}$ journal and benefit from:}

- Convenient online submission

- Rigorous peer review

- Open access: articles freely available online

- High visibility within the field

- Retaining the copyright to your article

Submit your next manuscript at $>$ springeropen.com 